Pacific Journal of Mathematics

SCHAUDER ESTIMATES UNDER INCOMPLETE HÖLDER

Paul Chase Fife 


\title{
SCHAUDER ESTIMATES UNDER INCOMPLETE HÖLDER CONTINUITY ASSUMPTIONS
}

\author{
PaUl Fife
}

Dedicated to Charles Loewner on the occasion of his 70th birthday

1. Introduction. In 1934 Schauder [6], [7] obtained a priori pointwise estimates for solutions to general second order linear elliptic differential equations. These estimates have been generalized and simplified by many authors, but by far the most general estimates of this type so far are the interior estimates of Douglis and Nirenberg [3] and the estimates up to the boundary of Agmon, Douglis, and Nirenberg [2]. In the latter paper the boundary-value problem

$$
\begin{aligned}
& L(x, D) u=f \text { in a domain } \mathscr{D}, \\
& B_{j}(x, D) u=\varphi_{j} \text { on a portion of the boundary } \\
& \qquad \dot{\mathscr{D}}(j=1,2, \cdots, m),
\end{aligned}
$$

is considered, where $L$ is uniformly elliptic of order $2 m$ and the $B_{j}$ satisfy the "complementing condition" with respect to $L$. Roughly speaking, under certain smoothness assumptions on the coefficients of $L$ and $B_{j}$, on $\dot{\mathscr{D}}$, and on the functions $u, f, \varphi_{j}$, a priori bounds on certain derivatives of $u$ and their Hölder difference quotients are obtained in terms of the maximum values in $\mathscr{D}$ (or $\dot{\mathscr{D}}$ ) of certain derivatives of $f$ and $\varphi_{j}$ and their Hölder difference quotients. As a byproduct at one stage near the beginning, an estimate is obtained (their Theorem 2.2) for the case of constant coefficients and a halfspace domain, in which no Hölder difference quotients occur. This estimate leads to a maximum principle. The history of this latter kind of estimate is also extensive, but maximum principles of greatest generality seem to have been obtained by Agmon [1].

The present paper explores the possibility of obtaining a priori pointwise estimates involving Hölder difference quotients not with respect to all, but only with respect to some of the independent variables $x_{i}$. With a few exceptions, the argument follows in basic outline the argument in [2]. Also the notation of [2] is preserved where possible. Throughout the paper $n+1$ denotes the number of independent variables, and $q$ of them $(0 \leqq q \leqq n+1)$ are distinguished from the others in that relevant functions are considered to be Hölder

Received May 31, 1962, and in revised form January 30, 1963. This work was supported in part by Office of Naval Research Contract Nonr-225 (11) at Stanford University. Reproduction in whole or in part is permitted for any purpose of the United States Government. 
continuous only in the distinguished variables.

The first step is the derivation of certain potential theoretic results in $\S 2$. Results of this nature go back to Hjlder, Petrini, Korn, and Lichtenstein (see the survey in [5]). These are applied in $\S 3$ to functions given by convolutions with a fundamental solution to an elliptic operator as kernel, and in $\S 4$ to solutions of the basic boundary value problem with compact support when the operators have constant coefficients and $\mathscr{D}$ is a half-space. These results are in the form of sufficient conditions on the operator $P(D)$ in order that $P(D) u$ may be estimated in terms of certain derivatives and "distinguished" Hölder difference quotients of $L u$ and $B_{j} u$. Also a necessary condition on $P(D)$ for such estimates to hold is given. Let $\hat{L}$ and $\hat{B}_{j}$ denote the operators obtained from $L$ and $B_{j}$ respectively by deleting all differentiations with respect to distinguished variables, and $\hat{u}$ a solution to the basic boundary-value problem with $L$ and $B_{j}$ replaced by $\hat{L}$ and $\hat{B}_{j}$. As a corollary it is found (in the constant coefficient, half-space case) that $u$ and $\hat{u}$ differ by a function whose appropriate derivatives have estimable Hölder difference quotients in all variables.

In $\S \S 5$ and 6 the results are extended to a class of problems with variable coefficients and domains with curved boundaries by the method [2,3]. The distinguished variables are now certain local curvilinear coordinates. When $q<n$ this method appears to be inapplicable to the general class treated in [2, §7]; in addition to the assumptions made there, we must impose the requirement that coordinate transformations exist which map small neighborhoods adjoining $\dot{\mathscr{D}}$ into hemispheres and which transform $L$ and $B_{j}$ into operators $L^{\prime}$ and $B_{j}^{\prime}$ such that, on the flat boundary of the hemisphere, $\hat{L}^{\prime}(x, D)$ $=\lambda(x) \hat{L}_{0}(D)$ and $\hat{B}_{j}^{\prime}(x, D)=\beta_{j}(x) \hat{B}_{j_{0}}(D)$ (the notation $\hat{L}^{\prime}, \hat{B}_{j}^{\prime}$ is explained above). In $\S 6$ the case $q=n$ is given special attention. It is shown that essentially every result in the area of the usual Schauder estimates $(q=n+1)$; i.e., every result in $\S \S 1-7$ of [2], has its analog with $q=n$. In particular, existence and uniqueness occurs in the classes of functions corresponding to $q=n$ exactly when it occurs in the classes corresponding to $q=n+1$. In $\S \S 5$ and 6 the coefficients in the operators $L$ and $B_{j}$ are assumed to be completely Hölder continuous.

The author expresses his gratitude to Professor L. Nirenberg for his suggestions.

2. Potential theory. Let $x$ be a point in $n$-space. We shall distinguish its first $q(0 \leqq q \leqq n)$ from its last $n-q$ coordinates and write $x=(\widetilde{x}, \hat{x})$, where $\tilde{x}=\left(x_{1}, \cdots, x_{q}\right)$ and $\hat{x}=\left(x_{q+1}, \cdots, x_{n}\right)$. If $q=n$ we write $x=\widetilde{x}$, and if $q=0, x=\widehat{x}$. The concern in this 
section will be with functions $u(x, t)$ defined in the $(n+1)$-dimensional half-space $t>0$ by a singular integral

$$
u(x, t)=\int K(x-y ; t) g(y) d y .
$$

In certain cases $u$ may be extended to be a continuous function in the closed half-space $t \geqq 0$; then we shall use the notation $u(x, 0)$ without further explanation. Our object is to exhibit conditions on the kernel $K$ under which certain boundedness and/or continuity properties of $u$ will be implied by similar properties of $g$.

Explicitly, we assume $K(x ; t)$ to be continuous except for $x=t$ $=0$, and that there is a constant $C_{1}$ such that

$$
D^{\mu} K(x ; t)>C_{1}\left(|x|^{2}+t^{2}\right)^{-(1 / 2)(n+\mu)} \quad(\mu=0,1)
$$

where here and below $D^{\mu}$ denotes any $\mu$ th order derivative. We also assume that

$$
\left|\int_{\tilde{y}-\text { space }} K(y ; t) d \widetilde{y}\right| \leqq C_{2} t\left(|\widehat{y}|^{2}+t^{2}\right)^{-(1 / 2)(n-q+1)}
$$

if $1 \leqq q \leqq n-1$,

$$
|K(\hat{x} ; t)| \leqq C_{2} t\left(|\hat{x}|^{2}+t^{2}\right)^{-(1 / 2)(n+1)}
$$

if $q=0$, and

$$
\left|\int_{|y|>\delta} K(y ; t) d y\right| \leqq C_{2} \quad \text { for all } \delta>0
$$

if $q=n$. In certain important cases the integral in (2.3a) will vanish; then we shall simply say that $C_{2}=0$.

Concerning $g(x)$ we assume that it is in $L_{\infty}$, has compact support, and is uniformly Hölder continuous for some exponent $\alpha(0<\alpha<1)$ with respect to the variables $\tilde{x}$ (in case $q>0$ ); i.e.,

$$
\operatorname{lub}_{\hat{x}, \widetilde{x} \widetilde{x}^{\prime}} \frac{\left|g(\widetilde{x}, \hat{x})-g\left(\widetilde{x}^{\prime}, \hat{x}\right)\right|}{\left|\widetilde{x}-\widetilde{x}^{\prime}\right|^{\alpha}}<\infty .
$$

It will be convenient to use the norm

$$
\begin{aligned}
{[g]_{x}^{q} } & =\text { true } \max |g|+\text { the above 1.u.b. for } q>0 ; \\
& =\text { true } \max |g| \text { for } q=0 .
\end{aligned}
$$

THEOREM 2.1. Under these assumptions the norm $[u]^{q}$ exists for all $t \geqq 0$ and

$$
[u]_{\infty}^{q}<C_{3}[g]_{\alpha}^{q}, \quad 0 \leqq q \leqq n,
$$


where $C_{3}$ depends only on $C_{1}, C_{2}, n, q$, and $\alpha$.

If in addition $C_{2}=0$, then $u(x, t)$ is Hölder continuous in all variables including $t$, and

$$
\operatorname{l.u}_{x, x^{\prime}, b_{t} t^{\prime}} \frac{\left|u(x, t)-u\left(x^{\prime}, t^{\prime}\right)\right|}{\left(\left|x-x^{\prime}\right|^{2}+\left|t-t^{\prime}\right|^{2}\right)^{\alpha / 2}}=[u]_{\alpha} \leqq C_{3}[g]_{\alpha}^{q} .
$$

This theorem, in the case $q=n$, yields the results proven in $[2$, $\S 3]$ (under slightly different hypotheses on $K$ ). Its proof is trivial in the case $q=0$, so we assume $q>0$. We shall employ the representation

$$
\begin{aligned}
u(x, t)= & \int d \hat{y} \int K(x-y ; t)[g(y)-g(\widetilde{x}, \hat{y})] d \widetilde{y} \\
& +\int d \hat{y} g(\widetilde{x}, \hat{y}) \int K(x-y ; t) d \widetilde{y}
\end{aligned}
$$

which is equivalent to (2.1). If $q=n$ it is understood that the symbols $\int d \hat{y}$ and $\hat{y}$ are to be omitted where they occur. Let $x=$ $(\widetilde{x}, \hat{x})$ and $x^{\prime}=\left(\widetilde{x}^{\prime}, \hat{x}^{\prime}\right)$ be any two points in $x$-space. Let $\delta=\left|x-x^{\prime}\right|$, $S$ the set of points $y$ with $\left|\widetilde{y}-\widetilde{x}^{\prime}\right|<2 \delta,\left|\hat{y}-\hat{x}^{\prime}\right|<2 \delta$, and $E$ the exterior of $S$. Then using (2.7) we write

$$
u(x, t)-u\left(x^{\prime}, t\right)=I_{1}+\cdots+I_{7},
$$

where

$$
\begin{aligned}
& I_{1}=\int_{S} K(x-y ; t)[g(y)-g(\widetilde{x}, \hat{y})] d y, \\
& I_{2}=-\int_{S} K\left(x^{\prime}-y ; t\right)\left[g(y)-g\left(\widetilde{x}^{\prime} ; y\right)\right] d y, \\
& I_{3}=\int_{E}\left[K(x-y ; t)-K\left(x^{\prime}-y ; t\right)\right][g(y)-g(\widetilde{x}, \hat{y})] d y, \\
& I_{4}=-\int_{\left|\hat{y}-\hat{x}^{\prime}\right|>2 \delta} d \hat{y}[g(\widetilde{x}, \hat{y})-(\widetilde{x}, \hat{y})] \int K\left(x^{\prime}-y ; t\right) d \widetilde{y}, \\
& I_{5}=-\int_{\left|\hat{y}-\hat{x}^{\prime}\right|<2 \delta} d \hat{y}\left[g\left(\widetilde{x}^{\prime}, \hat{y}\right)-g(\widetilde{x}, \hat{y})\right] \int_{\left|\widetilde{y}-\widetilde{x}^{\prime}\right|>2 \delta} K\left(x^{\prime}-y ; t\right) d \widetilde{y}, \\
& I_{6}=\int d \hat{y}\left[g(\widetilde{x}, \hat{y})-g\left(\widetilde{x}^{\prime}, \hat{y}\right)\right] \int K(x-y ; t) d \widetilde{y}, \\
& I_{7}=\int d \hat{y} g\left(\widetilde{x}^{\prime}, \hat{y}\right) \int\left[K(x-y ; t)-K\left(x^{\prime}-y ; t\right)\right] d \widetilde{y} .
\end{aligned}
$$

In case $q=n$ we set $I_{4}=0$ and disregard the integration with respect to $\hat{y}$ in $I_{5-7}$.

Since $|g(y)-g(\widetilde{x}, \hat{y})| \leqq[g]_{\alpha}^{q}|\widetilde{y}-\widetilde{x}|^{\alpha} \leqq[g]_{\alpha}^{\alpha}|y-x|^{\alpha}$, it follows that $\left|I_{3}\right|<$ const. $[g]_{\alpha}^{\alpha} \delta^{\alpha}$. Using (2.2) again we see by the usual argument that $I_{1}$ and $I_{2}$ are subject to the same estimate. $I_{4}$ and $I_{6}$ may be 
estimated by (2.3a):

$$
\left|I_{4}\right|,\left|I_{6}\right| \leqq C_{2}[g]_{\alpha}^{q} \delta^{\alpha} t \int\left(|\hat{y}|^{2}+t^{2}\right)^{(1 / 2)(-n+q-1)} d \hat{y} \leqq \text { const. }[g]_{\alpha}^{\alpha} \delta^{\alpha}
$$

To estimate $I_{5}$ we set $r=\left|\widetilde{y}-\widetilde{x}^{\prime}\right|$ so that $\left|K\left(x^{\prime}-y\right)\right|<C_{1} r^{-n}$, and obtain, if $q<n,\left|I_{5}\right| \leqq$ cont. $[g]_{\alpha}^{q} \delta^{\alpha} \int_{\left|\hat{y}=\hat{x}^{\prime}\right|<2 \delta} \int_{\delta}^{\infty} r^{-n+q-1} d r \leqq$ const. [g $]_{\alpha}^{q} \delta^{\alpha}$. If $q=n$ we use (2.3c) to obtain the same estimate.

The estimates obtained so far tell us that

$$
\left|u(x, t)-u\left(x^{\prime}, t\right)\right|<\text { const. }[g]_{\alpha}^{q}\left|x-x^{\prime}\right|^{\alpha}+\left|I_{7}\right| .
$$

Now $I_{7}$ will vanish provided that either $(a) C_{2}=0$, or (b) $x$ and $x^{\prime}$ differ only in their first $q$ components; i.e., $x=(\widetilde{x}, \hat{x}), x^{\prime}=\left(\widetilde{x}^{\prime}, \widehat{x}\right)$. Condition (b) is sufficient because

$$
\begin{aligned}
& \int\left[K(x-x ; t)-K\left(x^{\prime}-y ; t\right) d \widetilde{y}=\int K(\widetilde{x}-\widetilde{y}, \hat{x}-\hat{y} ; t) d \widetilde{y}\right. \\
& \quad-\int K\left(\widetilde{x}^{\prime}-\widetilde{y}, \widehat{x}-\hat{y} ; t\right) d \widetilde{y}=0 .
\end{aligned}
$$

Now assume condition (b) to hold, so that the last term in (2.8) does not appear. Taking the l.u.b. of the left side, (2.5) is proven for the case $1 \leqq q \leqq n$. It is easily extended, however, to the case $q=0$ by using (2.1) and (2.3b).

To prove the second part of Theorem 2.1 we assume condition (a); i.e., $C_{2}=0$, so that again the last term in (2.8) disappears. The only thing left to prove is Hölder continuity with respect to $t$. Let $t, t^{\prime}$ be two numbers such that $0 \leqq t<t^{\prime}$. Since the last integral in (2.7) also vanishes we may write

$$
u\left(x, t^{\prime}\right)-u(x, t)=\int d \widetilde{y} \int\left(\int_{t}^{t^{\prime}} K_{t}(x-y ; \tau) d \tau\right)[g(y)-g(\widetilde{x}, \hat{y})] d \hat{y} .
$$

Again (2.2) tells us that this integral is absolutely convergent, so we write it as

$$
\int_{t}^{t^{\prime}} \int_{\mathrm{al1} y} K_{t}(x-y ; \tau)[g(y)-g(\widetilde{x}, \hat{y})] d y d \tau=I_{8}+I_{9},
$$

where

$$
I_{8}=\int_{t}^{t^{\prime}} \int_{|x-y|<t^{\prime}-t} \cdots d y d \tau
$$

and

$$
I_{9}=\int_{t}^{t^{\prime}} \int_{|x-y|>t^{\prime}-t} \cdots d y d \tau
$$


Setting $\rho^{2}=|x-y|^{2}+\tau^{2}$, we may estimate

$$
\begin{aligned}
\left|I_{8}\right| & \leqq \text { const }[g]_{\alpha}^{q} \int_{t}^{2 t^{\prime}-t} \rho^{-1+\alpha} d \rho \leqq \text { const }[g]_{\alpha}^{\alpha}\left[\left(t^{\prime}\right)^{\alpha}-t^{\alpha}+\left(t^{\prime}-t\right)^{\alpha}\right] \\
& \leqq \text { const }[g]_{\alpha}^{q}\left|t^{\prime}-t\right|^{\alpha}
\end{aligned}
$$

and

$$
\left|I_{9}\right| \leqq \text { const }[g]_{\alpha}^{q}\left|t^{\prime}-t\right| \int_{t^{\prime}-t}^{\infty} r^{-2+\alpha} d r \leqq \text { const }[g]_{\alpha}^{q}\left|t^{\prime}-t\right|^{\alpha} .
$$

Combining these results with (2.8), (2.6) is easily obtained, completing the proof of Theorem 2.1.

Since the above constants do not depend on $t$ or $t^{\prime}$, this last argument yield an immediate corollary:

COROLLARY 2.1: Let

$$
\bar{U}(x, t)=\int d \hat{y} \int K(x-y ; t)[g(y)-g(\widetilde{x}, \widehat{y})] d \widetilde{y},
$$

the first term in (2.7). Then $\bar{U}$ may be extended as a completely Hölder-continuous function to the closed region $t \geqq 0$, in which it satisfies the estimate (2.6).

3. Interior-type estimates. In using Theorem 2.1 to obtain Schauder estimates the kernel $K$ will be interpreted as a derivative of a fundamental solution or of a Poisson kernel for an elliptic boundary value problem. In this section we treat the case when $K$ is a derivative of a fundamental solution.

The following norms and pseudonorms will be employed extensively. They refer to functions defined in the half-space $t>0$ (or on the hyperplane $t=0$ ). The differentiability properties needed for the quantities below to be well-defined will be obvious. These norms and pseudonorms will correspond to those in [2, §5]. Subscripts will always denote the order of differentiation, and superscripts the independent variables with respect to which the Hölder difference quotients are to be taken.

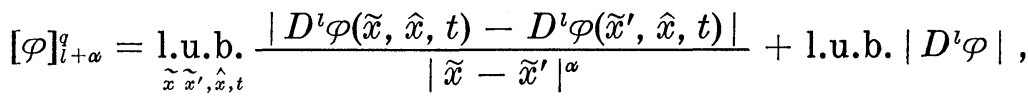

$$
\begin{aligned}
& {[\varphi]_{i+\alpha}^{q, t}=\underset{\widetilde{x}, \tilde{x}^{\prime}, \hat{x}, t, t^{\prime}}{\operatorname{lou}} \frac{\left|D^{l} \varphi(\widetilde{x}, \hat{x}, t)-D^{l} \varphi\left(\widetilde{x}^{\prime}, \hat{x}, t^{\prime}\right)\right|}{\left(\left|t-t^{\prime}\right|^{2}+\left|\widetilde{x}-\widetilde{x}^{\prime}\right|^{2}\right)^{\alpha / 2}}+\text { l.u.b. }\left|D^{l} \varphi\right| \text {, }}
\end{aligned}
$$

where, as before, $\widetilde{x}=\left(x_{1}, \cdots, x_{q}\right)$. In particular

$$
[\varphi]_{l+\alpha}^{0, t}=\lim _{x, t, t^{\prime}} \frac{\left|D^{\imath} \varphi(x, t)-D^{\imath} \varphi\left(x, t^{\prime}\right)\right|}{\left|t-t^{\prime}\right|^{\alpha}}+\text { l.u.b. }\left|D^{\imath} \varphi\right|,
$$




$$
[\varphi]_{l+\alpha}^{0}=\text { l.u.b. }\left|D^{\imath} \varphi\right|
$$

and

$$
[\varphi]_{l+\alpha}^{n t}=[\varphi]_{l+\alpha}^{n+1}=[\varphi]_{l+\alpha}
$$

in the sense the latter is used in [2], for instance. Of course, in all of these the l.u.b. is taken over all derivatives of order $l$. Also we define

$$
\begin{aligned}
& |\varphi|_{l+\alpha}^{q t}=\sum_{j=0}^{l} \text { l.u.b. }\left|D^{j} \varphi\right|+[\varphi]_{l+\alpha}^{q t}, \\
& |\varphi|_{l+\infty}^{q}=\sum^{l} \text { l.u.b. }\left|D^{j} \varphi\right|+[\varphi]_{l+\infty}^{q} .
\end{aligned}
$$

Corresponding to these norms we define $\mathscr{C}_{l+\infty}^{q}$ as the class of functions $\varphi$ defined in the half-space $t>0$ with continuous and bounded derivatives of order $<l$, and piecewise continous and bounded derivatives of order $l$ which are uniformly Hölder continuous in $\widetilde{x}$. The class $\mathscr{C}_{i+\infty}^{q t}$ has an analogous definition.

The symbol $\widetilde{D}^{\lambda}$ will denote any derivative of order $\lambda$, at least one of whose differentiations is with respect to a component of $\widetilde{x}$; i.e., $\widetilde{D}^{\lambda}=\left(\partial / \partial x_{i}\right) D^{\lambda-1}$, where $i \leqq q$.

REMARK: Let $\lambda$ be any integer $\geqq 1$. Assume $f(x)$ has absolutely continuous derivatives of order $\lambda-1$, that $q>0$, and that $[f]_{\lambda+\infty}^{\tau}$ is finite. Then every derivative $\widetilde{D}^{\lambda} f$ is Hölder continuous with respect to all variables, and

$$
\left[\widetilde{D}^{\lambda} f\right]_{\alpha}^{n} \leqq C(\alpha)[f]_{\lambda+\alpha}^{q},
$$

where $C$ depends only on $\alpha$.

Proof. It is sufficient to consider the case $\lambda=1, q=1, n=2$, for the general case may be reduced to this case by freezing all but two of the independent variables and replacing $f$ in the proof by some $D^{\lambda-1} f$. By assumption, then $\widetilde{x}$ and $\hat{x}$ have single components; call them $x$ and $y$ for simplicity, so that $f=f(x, y)$. The absolute continuity guarantees the identity

$$
\int_{x}^{x+h}\left[f_{x}(\xi, y+k)-f_{x}(\xi, y)\right] d \xi=\int_{y}^{y+k}\left[f_{y}(x+h, \eta)-f_{y}(x, \eta)\right] d \eta
$$

to hold for all values of $x, y, h$, and $k$. It follows that

$$
\begin{aligned}
h\left[f_{x}(x, y+k)-f_{x}(x, y)\right] & =\int_{y}^{y+k}\left[f_{y}(x+h, \eta)-f_{y}(x, h)\right] d \eta \\
& -\int_{x}^{x+h}\left[f_{x}(\xi, y+k)-f_{x}(x, y+k)\right] d \xi+
\end{aligned}
$$




$$
\int_{x}^{x+h}\left[f_{x}(\xi, y)-f_{x}(x, y) d \xi\right.
$$

The first term on the right is bounded in absolute value by $k h^{\alpha}[f]_{1+\alpha}^{1}$, and each of the other two by

$$
[f]_{1+\alpha}^{1} \int_{0}^{h}\left(\xi^{\prime}\right)^{\alpha} d \xi^{\prime}=\frac{1}{1+\alpha} h^{1+\alpha}[f]_{1+\alpha}^{1} .
$$

Dividing through by $h k^{\alpha}$ and setting $\sigma=h / k$, we have the estimate

$$
\left|f_{x}(x, y+k)-f_{x}(x, y)\right| k^{-\alpha} \leqq[f]_{1+\alpha}^{1}\left(\sigma^{\alpha-1}+\frac{2}{1+\alpha} \sigma^{\alpha}\right)
$$

for all values of $\sigma$. Taking the l.u.b. of the left over all $x, y$, and $k$, and the g.l.b. of the right over $\sigma$, we have $\left[f_{x}\right]_{x}^{2} \leqq C(\alpha)[f]_{1+\alpha}^{1}$. As mentioned, this generalizes immediately to (3.2).

The following lemma will constitute an application of Theorem 2.1 to the case when $K(x-y ; 0)$ is a fundamental solution of an elliptic differential operator in the variables $x$ with constant coefficients, and containing only derivatives of order $2 \mathrm{~m}$. The constant $H$ will be defined as an upper bound for the ellipticity constant of $L$, and for the coefficients of $L$. It is shown in [4] that a fundamental solution $\Gamma(x)$ to $L$ always exists having the property

$$
\left|D^{k} \Gamma(x)\right|<\mathrm{const}|x|^{2 m-n-k}(1+|\log | x||),
$$

the log term being omitted unless $n$ is even and $0 \leqq k \leqq 2 m-n$.

Theorem 3.1. Assume $1 \leqq q \leqq n$. Let $l$ be any number $\geqq 2 m$, and let $f(x)$ have derivatives of order $l-2 m$ which are uniformly Hölder continuous with respect to $\widetilde{x}$. If $l>2 m$ we also assume the derivatives of order $l-2 m-1$ to be absolutely continuous, and if $l=2 m, f(x)$ is to be integrable. (That derivatives $D^{l-2 m} f$ are integrable for $l>2 m$ follows from the absolute continuity assumption.) Also we assume $f$ to have compact support. Then if

$$
v(x)=\int \Gamma(x-y) f(y) d y,
$$

every derivative $\widetilde{D}^{l} v$ exists and

$$
\left[\widetilde{D}^{l} v\right]_{\alpha}^{n} \leqq \text { const }[f]_{l-2 m+\alpha}^{q} .
$$

The constants here depend only on $H, n, m, l$, and $\alpha$.

Proof. The case $q=n$ is a well-known result, so we take $0 \leqq$ $q \leqq n-1$. Differentiating equation (3.4) $l-1$ times while integrating by parts if necessary we have 


$$
\widetilde{D}^{l-1} v=\int \widetilde{D}^{2 m-1} \Gamma(x-y) D^{l-2 m} f(y) d y .
$$

Now let $x^{\prime}$ be a point, all except one of whose coordinates are the same as those of $x$. We shall derive the following representation for the corresponding difference quotient:

$$
\begin{aligned}
& \frac{\widetilde{D}^{l-1} v(x)-\widetilde{D}^{l-1} v\left(x^{\prime}\right)}{\left|x-x^{\prime}\right|} \\
& \quad=\int \frac{\widetilde{D}^{2 m-1} \Gamma(x-y)-\widetilde{D}^{2 m-1} \Gamma\left(x^{\prime}-y\right)}{\left|x-x^{\prime}\right|}\left[D^{l-2 m} f(y)\right. \\
& \left.-D^{l-2 m} f(\widetilde{x}, \hat{y})\right] d y .
\end{aligned}
$$

Let $x_{j}$ be the component of $\widetilde{x}$ with respect to which a differentiation occurs in the operator $\widetilde{D}^{2 m-1}$ in $(3.6 \mathrm{a})$, so that $\widetilde{D}^{2 m-1}=\left(\partial / \partial x_{j}\right) D^{2 m-2}$. Then, since $D_{x} \Gamma(x-y)=-D_{y} \Gamma(x-y)$,

$$
\begin{aligned}
\int_{-\infty}^{\infty}[ & \left.\widetilde{D}^{2 m-1} \Gamma(x-y)-\widetilde{D}^{2 m-1} \Gamma\left(x^{\prime}-y\right)\right] d y_{j} \\
= & -\lim _{y_{j} \rightarrow \infty}\left[D^{2 m-2} \Gamma(x-y)-D^{2 m-2} \Gamma\left(x^{\prime}-y\right)\right] \\
& \quad+\lim _{y_{j} \rightarrow-\infty}\left[D^{2 m-2} \Gamma(x-y)-D^{2 m-2} \Gamma\left(x^{\prime}-y\right)\right]=0,
\end{aligned}
$$

as can be seen from the behavior of $\Gamma$ at infinity indicated in (3.3) (using also the mean value theorem in the case $n=2$ ). It follows immediately that

$$
\begin{aligned}
& \frac{\widetilde{D}^{l-1} v(x)-\widetilde{D}^{l-1} v\left(x^{\prime}\right)}{\left|x-x^{\prime}\right|} \\
& =\int^{\prime} d y \int \frac{\widetilde{D}^{2 m-1} \Gamma(x-y)-\widetilde{D}^{2 m-1} \Gamma\left(x^{\prime}-y\right)}{\left|x-x^{\prime}\right|} \\
& {\left[D^{l-2 m} f(y)-D^{l-2 m} f(\widetilde{x}, \hat{y})\right] d y_{j},}
\end{aligned}
$$

where $\int^{\prime} d y$ signifies integration with respect to all variables except $y_{j}$. But this integral is absolutely convergent, as can be seen by applying the mean value theorem to the difference quotient in the integral, using (3.3), and recognizing that the integrations with respect to components of $\widetilde{y}$ may be considered as only over a finite range (since $f(y)$ has compact support); the order of integration is therefore immaterial and (3.6b) is valid. Defining $D^{\prime}$ as the derivative in the direction from $x$ to $x^{\prime}$ and $\widetilde{D}^{2 m}=D^{\prime} \widetilde{D}^{2 m-1}$, we subtract the absolutely convergent integral

$$
\int \widetilde{D}^{2 m} \Gamma(x, y)\left[D^{l-2 m} f(y)-D^{l-2 m} f(\widetilde{x}, \widehat{y})\right] d y
$$


from each side of (3.6b), obtaining on the right an integral which is bounded in absolute value by const. $\left|x-x^{\prime}\right|{ }^{\alpha}[f]_{l-2 m+\alpha}^{q}$. This last estimate is obtained by the usual process of splitting the region of integration into the sphere $|y-x|<2\left|x-x^{\prime}\right|$ and its exterior, and applying the mean value theorem in the latter region. Now letting $x^{\prime} \rightarrow x$, this bound vanishes, and furthermore the left side of (3.6b) approaches $\widetilde{D}^{l} v(x)$. Hence

$$
\widetilde{D}^{\imath} v=\int \widetilde{D}^{2 m} \Gamma(x-y)\left[D^{\imath-2 m} f(y)-D^{\imath-2 m} f(\widetilde{x}, \widehat{y})\right] .
$$

This integral is reminiscent of the first term on the right of (2.7); and in fact we shall apply Corollary 2.1 directly in proving the theorem. We identify $K(x-y ; 0)$ with $\widetilde{D}^{2 m} \Gamma(x-y)$ and $g(y)$ with $D^{l-2 m}$ $f(y)$; then according to Corollary 2.1, (3.5) will follow from (2.6) if the hypotheses (2.2) and (2.3a) with $t=0$ are true. But (2.2) follows from (3.3) and (2.3a) from our representation of $K$ as a derivative. Theorem 3.1 is thereby proved.

4. Boundary-type estimates. In this section $L(D)$ will again be an elliptic differential operator with constant coefficients containing only terms of order $2 m$; but now it will be an operator in the $n+1$ variables $x_{1}, \cdots, x_{n}, t$. Similarly, let $B_{j}(D)(j=1, \cdots, m)$ be operators with constant coefficients and only terms of order $m_{j}$. We assume $L$ and $B_{j}$ to satisfy the root condition and complementing condition stated in $[2, \S 1]$. The concern here will be with the boundary-value problem

$$
\begin{aligned}
L\left(D_{x}, D_{t}\right) u & =f(x, t) & & (t>0) \\
B_{j}(D) u & =\varphi_{j}(x) & & (t=0, j=1, \cdots, m) .
\end{aligned}
$$

We initially assume all functions to be infinitely differentiable and to have compact support; this restiction will be removed at the end of the section (Theorem 4.6).

First we review some important results from [2] concerning representations of the function $u(x, t)$. Let $l$ be any integer with $l \geqq \max \left(2 m, m_{j}\right)$, and $P(D)$ a differential operator, each term of which is of degree $l$. Then

$$
\begin{aligned}
& P(D) u(x, t)=P(D) v(x, t) \\
& \quad+\sum_{j=1}^{m} \int P(D) K_{j}(x-y ; t)\left[\varphi_{j}(y)-\psi_{j}(y)\right] d y,
\end{aligned}
$$

where $v(x, t)=\int \Gamma(x-y, t-\tau) f_{N}(y, \tau) d y d \tau, \Gamma(x-y, t-\tau)$ is a fundamental solution for $L, f_{N}$ is a sufficiently smooth extension of $f(x, y)$ 
to the whole space such that $f_{N}$ has compact support, $\psi_{j}(x)=$ $\left.B_{j}(D) v(x, t)\right|_{t=0}$ and $K_{j}$ are Poisson kernels given explicitly in [2].

Section 3 was concerned with estimating the first term on the right of (4.2) in terms of properties of $f$. We shall now consider the other terms and develop estimates for functions given by

$$
w(x, t)=\int K_{j}(x-y ; t) \varphi_{j}(y) d y=K_{j} * \varphi_{j}
$$

It is proved in [2] that

$$
[w]_{l+\alpha}^{n, t} \leqq C\left[\varphi_{j}\right]_{l-m,+\alpha}^{n} . \quad\left(l \geqq m_{j}\right)
$$

(for the notation see (3.1)). Also it is proved in [2] that

$$
[P(D) w]_{0}^{0} \leqq C\left[\varphi_{j}\right]_{l-m j}^{0}
$$

provided $P(\xi, \tau)$ (obtained from $P(D)$ by replacing $\partial / \partial x_{i}$ by $\xi_{i}$ and $\partial / \partial t$ by $\tau)$ is of the form

$$
P(\xi, \tau)=\sum_{j=1}^{m} \xi^{l-m_{J}} B_{j}(\xi, \tau),
$$

where $\xi^{l-m_{j}}$ stands for any monomial of degree $l-m_{j}$ in the variables $\xi_{i}$ alone.

(4.4) corresponds to the case $q=n$; (4.5) to the case $q=0$. Our primary aim in this section will be to supplement these estimates by (1) extending them to intermediate values of $q, 0<q<n$, and (2) deriving, for $q<n$, a necessary condition on $P(\xi, \tau)$ for such estimates to hold.

First we shall review and develop certain properties of the Poisson kernels. The kernels are given by

$$
\begin{aligned}
& K_{j}(x ; t)=\Delta_{x}^{(n+s) / 2} K_{j, s}(x, t), \\
& K_{j, s}(x ; t)=b_{j, s} \int_{|\xi|=1} \int_{\gamma} \frac{N_{j}(\xi, \tau)(x \cdot \xi+t \tau)^{m_{j+s}}}{M^{+}(\xi, \tau)} \\
&\left(\log \frac{x \cdot \xi+t \tau}{i}+c_{j, s}\right) d \tau .
\end{aligned}
$$

Here $b_{j, s}$ and $c_{j, s}$ are appropriate constants; $M^{+}(\xi, \tau)=\prod_{k=1}^{m}\left(\tau-\tau_{k}^{+}(\xi)\right)$ where $\tau_{k}^{+}(\xi), k=1, \cdots, m$ are the $m$ roots of $L(\xi, \tau)=0$ with positive imaginary part $\left(L(\xi, \tau)\right.$ is the polynomial obtained by replacing $\partial / \partial x_{i}$ by $\xi_{i}$ and $\partial / \partial t$ by $\tau$ in $L\left(D_{x}, D_{t}\right)$ ); the contour $\gamma$ surrounds the $m$ roots $\tau_{k}^{+}(\xi)$ and lies entirely above the real axis; $N_{j}(\xi, \tau)$ are polynomials in $\tau$ such that

$$
\int_{\gamma} \frac{N_{j}(\xi, \tau) B_{k}(\xi, \tau)}{M^{+}(\xi, \tau)} d \tau=\delta_{j k}
$$


In (4.6) and elsewhere below, if $n=1$ then $\int_{|\xi|=1} d w_{\xi}$ is to be understood as $\sum_{\xi= \pm 1}$.

We shall state three lemmas concerning integrals such as occur in (4.6).

LEMMA 4.1. Let $F(\xi)$ be a function of the real vector $\xi$ continuous on the sphere $|\xi|=1$. Let $\tau_{0}$ be a complex constant with Im $\tau_{0} \neq 0$, and $k$ an integer $\geqq 1$. Then

$$
\left|\int_{|\xi|=1} F(\xi)\left(x \cdot \xi+t \tau_{0}\right)^{-k} d \omega_{\xi}\right| \leqq C\left(|x|^{2}+t^{2}\right)^{-k / 2},
$$

$C$ depending on $\tau_{0}, k$, and $\max |F|$.

The proof of this lemma is given in Appendix 1 of [2]. This same estimate will clearly hold if the integrand is replaced by

$$
\int_{\gamma} F(\xi, \tau)(x \cdot \xi+t \tau)^{-k} d \tau,
$$

where $\gamma$ is a finite contour in the complex $\tau$-plane bounded away from the real axis, and $F(\xi, \tau)$ is continuous for $\tau \in \gamma,|\xi|=1$.

LEMMA 4.2. If $\lambda \geqq m_{j}+s+1$,

$$
\left|D^{\lambda} K_{j, s}\right|<C\left(|x|^{2}+t^{2}\right)^{(1 / 2)\left(m_{j}+s-\lambda\right)} \text {. }
$$

If $D_{x}^{\lambda}$ is any derivative of order $\lambda \geqq 0$ in the variables $x$, then

$$
\left|D_{x}^{\lambda} B_{k}(D) K_{j}(x ; t)\right|<C t\left(|x|^{2}+t^{2}\right)^{(1 / 2)\left(m_{j}-m_{k}-n-\lambda-1\right)} .
$$

If $k \neq j, \lambda \geqq m_{j}-m_{k}+s$, then

$$
\left|D_{x}^{\lambda} B_{k} K_{j, s}(x, t)\right|<C t\left(|x|^{2}+t^{2}\right)^{(1 / 2)\left(m_{j}-m_{k}+s-\lambda-1\right)} .
$$

In all these, $C$ depends only on the ellipticity constant, bounds for the coefficients in $L$ and $B_{j}$, the complementing condition constant, and all integers mentioned.

Proof. These estimates follow from Lemma 4.1 and the properties of $N_{j}$ and are given in [2] (eqs. $(2.13)^{\prime},(2.15)$ ).

LEMMA 4.3. Let the first $q(0 \leqq q \leqq n-1)$ coordinates of $n$-space be distinguished as in $\S 2$, and write $x=(\widetilde{x}, \widehat{x}), \xi=(\tilde{\xi}, \hat{\xi})$. Writing $L(\xi, \tau)=L(\tilde{\xi}, \hat{\xi}, \tau)$, let the polynomial $\hat{L}(\hat{\xi}, \tau)=L(0, \hat{\xi}, \tau)$, and similarly $\hat{B}_{i}(\xi, \tau)=B_{j}(0, \hat{\xi}, \tau)$. Let $\hat{K}_{j, s}$ be the Poisson kernels corresponding to $\hat{L}$ and $\hat{B}_{j}$ in $(n-q+1)$-space. Let $P(D)$ be a homogeneous differential operator of order $>m_{j}+s+q$ and $\hat{P}$ the operator 
obtained from $P$ by omitting all differentiations with respect to components of $\widetilde{x}$. Then

$$
\int_{\widetilde{x}-\text { space }} P(D) K_{j, s}(x ; t) d \widetilde{x}=\hat{P}(D) \hat{K}_{j, s}(\widehat{x} ; t) .
$$

This lemma is proved in Appendix A.

The following is an interesting consequence of Lemma 4.3 and the results of $\S 2$. In this and the other theorems of this section, $C$ denotes a constant depending only on the quantities listed in Lemma 4.2 .

THEOREM 4.1. Corresponding to the function $w(x)$ given by (4.3) define

$$
\hat{w}(x, t)=\hat{K}_{j} * \varphi_{j}(x)=\int_{\hat{y}-\text { space }} \hat{K}_{j}(\hat{x}-\hat{y}, t) \varphi_{j}(\widetilde{x}, \hat{y}) d \hat{y},
$$

so that $\widetilde{x}$ appears only as a parameter in the function $\varphi_{j}$. Also define $W(x, t)=w-\hat{w}$. Then if $l \geqq m_{j}$,

$$
[\widehat{W}]_{l+\alpha} \leqq c\left[\varphi_{j}\right]_{l-m_{j}+\alpha}^{q},
$$

where the symbol $\widehat{[.]_{l+\alpha}}$ is defined as is $[.]_{l+\alpha}^{n t}$, except that the quantity inside brackets is considered a function of $\hat{x}$ alone (and dependence on $\widetilde{x}$ is ignored).

This means that $w$ and $\hat{w}$ differ by a function whose appropriate derivatives have estimable Hölder difference quotients with respect to all $n-q+1$ variables $\hat{x}, t$. Actually the proof will show that only those derivatives whose order with respect to components of $\widetilde{x}$ is greater than $l-m_{j}$ need be excluded.

Proof. Let $D^{l}$ be any derivative of order $l$ in the variables $\hat{x}$ and $t$. We assume $l-m_{j}$ to be even; a similar proof goes through for the odd case. Applying (4.6) and integrating by parts, as is done in [2], we have

$$
D^{\imath} w=D^{l} K_{j} * \varphi_{j}=D^{l} \Delta^{(1 / 2)\left(n+s-l+m_{j}\right)} K_{j, s} * \Delta^{(1 / 2)(l-m \rho)} \varphi_{j} .
$$

From (4.8a) we know that (2.2) holds for the kernel $D^{l} \Delta^{(1 / 2)\left(n+s-l+m_{j}\right)} K_{j, s}$, so we may decompose the convolution into two terms as in (2.7):

$$
D^{\imath} w=I_{1}+I_{2}
$$

where

$$
\begin{aligned}
I_{1}= & \int d \widehat{y} \int D^{\imath} \Delta^{(1 / 2)\left(n+s-l+m_{\jmath}\right)} K_{j, s}(x-y ; t) \\
& \cdot\left[\Delta^{(1 / 2)\left(l-m_{j}\right)} \varphi_{j}(y)-\left.\Delta^{(1 / 2)\left(l-m_{j}\right)} \varphi_{j}(y)\right|_{\tilde{y}=\tilde{x}}\right] d \widetilde{y}
\end{aligned}
$$


and satisfies

$$
\left[I_{1}\right]_{\alpha}^{n t} \leqq C\left[\varphi_{j}\right]_{l-m g+\alpha}^{q}
$$

(according to Corollary 2.1); and

$$
I_{2}=\left.\left(\int_{\tilde{x}-\text { space }} D^{l} \Delta^{(1 / 2)(n+s-l+m \jmath)} K_{j, s} d \widetilde{x}\right) * \Delta^{(1 / 2)(l-m g)} \varphi_{j}(y)\right|_{\tilde{y}=\tilde{x}},
$$

which, according to Lemma 4.3 , is simply

$$
\left.D^{\imath} \hat{\Delta}^{(1 / 2)(n+s-l+m j)} \hat{K}_{j, s} * \Delta^{(1 / 2)(l-m))} \varphi_{j}\right|_{\tilde{y}=\tilde{x}}=I_{3}+I_{4},
$$

where

$$
\left.I_{3}=D^{l} \hat{\Delta}^{(1 / 2)(n+s-l+m}\right) \hat{K}_{j, s} * \widetilde{\Delta}^{(1 / 2)\left(l-m_{j}\right)} \varphi_{j}(\widetilde{x}, \hat{y}),
$$

and

$\left.I_{4}=D^{\imath} \hat{\Delta}^{(1 / 2)(n+s-l+m g}\right) \hat{K}_{j, s} * \hat{\Delta}^{(1 / 2)(l-m g)} \varphi_{j}(\widetilde{x}, \widehat{y})=D^{l} \hat{K}_{j} * \varphi_{j}(\widetilde{x}, \hat{y})=D^{l} \hat{w}(x, t)$,

the operators $\hat{\Delta}$ and $\widetilde{\Delta}$ denoting the Laplacian in $\hat{x}$ and $\widetilde{x}$ respectively. Now (3.2) yields the estimate

$$
\left[\widetilde{\Delta}^{(1 / 2)(l-m j)} \varphi_{j}(\widetilde{x}, \widehat{y})\right]_{\alpha}^{n} \leqq C\left[\varphi_{j}\right]_{i-m,+\alpha}^{q},
$$

hence the usual boundary estimates ([2], or Theorem 2.1 with $q=n$ ), indicate that

$$
\left[\hat{I}_{3}\right]_{\alpha} \leqq C\left[\varphi_{j}\right]_{l-m_{j}+\alpha}^{q} .
$$

But since $D^{\imath} W=I_{1}+I_{3}$, (4.10) is proven.

We are now ready to develop the two principal theorems of this section. The complementing condition states that for every $\xi \neq 0$, the $m$ operators $B_{j}(\xi, \tau)$ are, as polynomials in $\tau$, linearly independent. modulo $M^{+}(\xi, \tau)$. It follows that every polynomial $P(\xi, \tau)$ admits a. decomposition of the form

$$
P(\xi, \tau)=a(\xi, \tau) M^{+}(\xi, \tau)+\sum_{j=1}^{m} a_{j}(\xi) B_{j}(\xi, \tau),
$$

where $a(\xi, \tau)$ is a polynomial in $\tau$, but $a(\xi, \tau)$ and $a_{j}(\xi)$ are not. necessarily polynomials in $\xi$.

Theorem 4.2. (Sufficient condition.) Let the polynomial $P(\xi, \tau)$ be normalized and homogeneous of degree $l \geqq \max \left[m_{j}\right]$. Let $q$ be in

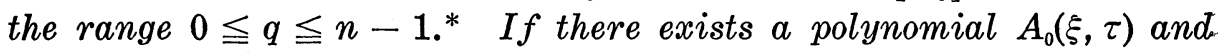
polynomials $a_{0 j}(\xi)$ (of degrees $l-m_{j}$ ) such that

\footnotetext{
* If $q=n$ we know from [2] that (4.16) holds for every $p$ of degree $l$.
} 


$$
P(0, \hat{\xi}, \tau)=A_{0}(\hat{\xi}, \tau) L(0, \hat{\xi}, \tau)+\sum_{j=1}^{m} a_{0 j}(\hat{\xi}) B_{j}(0, \hat{\xi}, \tau)
$$

then

$$
[P(D) w]_{\alpha}^{q} \leqq C\left[\varphi_{j}\right]_{l-m_{j}+\alpha}^{q} .
$$

THEOREM 4.3. (Necessary condition.) Again let $0 \leqq q \leqq n-1$. $A$ necessary condition on $P(\xi, \tau)$ (normalized and homogeneous of degree $l$ ) in order that the estimate (4.16) hold for all $\varphi_{j}$ infinitely differentiable and with compact support is that there exist a polynomial $A_{0}(\hat{\xi}, \tau)$ and functions $a_{0 j}(\hat{\xi}), 1 \leqq j \leqq m$, with $a_{0 j}(-\hat{\xi})=$ $(-1)^{l-m}{ }^{l} a_{0 j}(\hat{\xi})$ such that $(4.15)$ holds.

The difference between the two conditions is that only in the first case are the $a_{0 j}(\xi)$ assumed to be polynomials. The author is of the opinion that the condition in Theorem 4.2 is necessary as well as sufficient. Theorem 4.3 is proved in Appendix B.

Proof of Theorem 4.2. The case $q=0$ is essentially the abovementioned result (4.5) obtained in [2]. Therefore assume $1 \leqq q \leqq$ $n-1$. From (4.15) it follows that

$$
P(\xi, \tau)=A_{0}(\hat{\xi}, \tau) L(\xi, \tau)+\sum_{j=1}^{m} a_{0 j}(\hat{\xi}) B_{j}(\xi, \tau)+\widetilde{Q}(\xi, \tau),
$$

where $\widetilde{Q}$ is a polynomial every term of which contains as factor some component of $\tilde{\xi}$. We write

$$
P(D) w=W_{1}+W_{2},
$$

where (using (4.3), (4.6)),

$$
\begin{aligned}
& W_{1}=A_{0}\left(\hat{D}, D_{t}\right) L(D) K_{j} * \varphi_{j}+a_{0 j}(\hat{D}) B_{j} K_{j} * \varphi_{j}, \\
& W_{2}=\left(\sum_{i \neq j} a_{0 i}(\hat{D}) B_{j}+\widetilde{Q}(D)\right) \Delta^{(1 / 2)(n+s)} K_{j, s} * \varphi_{j} .
\end{aligned}
$$

(Here $s$ is an integer of the same parity as $n$ such that $n+s+m_{j}$ $-l>0$.) Since $L K_{j}=0$, we may write

$$
W_{1}=B_{j} K_{j} * a_{0 j}(\hat{D}) \varphi_{j} \text {. }
$$

Also, writing $R=\sum_{i \neq j} a_{0 i} B_{i}+\widetilde{Q}$, we follow the procedure in [2] and write

$$
W_{2}=R(D) \Delta^{(1 / 2)(n+s+m j-l)} K_{j, s} * \Delta^{(1 / 2)(l-m)} \varphi_{j}
$$

if $l-m_{j}$ is even, and

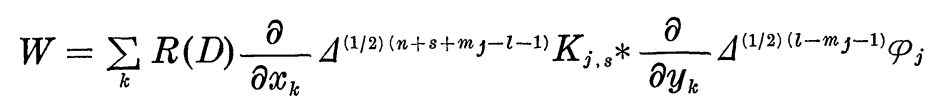


if $l-m_{j}$ is odd. For simplicity we consider only the even case. Theorem 2.1 may now be applied by identifying the $u$ in it with $W_{1}$ or $W_{2}, g$ with $a_{0 j} \varphi_{j}$ or $\Delta^{(1 / 2)\left(l-m_{j}\right)} \varphi_{j}$, and $K$ with $B_{j} K_{j}$ or $R \Delta^{(1 / 2)\left(n+s m_{j}-l\right)}$ $K_{j, s}$. Conditions (2.2) and (2.3a) must be verified. The first follows from (4.8a), and for (2.3a) we use Lemma 4.3 and (4.8b):

$$
\left|\int B_{j} K_{j} d \widetilde{x}\right|=\left|\hat{B}_{j} \hat{K}_{j}\right| \leqq C t\left(|\widehat{x}|^{2}+t^{2}\right)^{(1 / 2)(-n+q-1)} ;
$$

also, using (4.8c) and the fact that $\widetilde{Q}(0, \hat{\xi}, \tau)=0$,

$$
\begin{aligned}
& \left|\int R \Delta^{(1 / 2)\left(n+s+m_{j}-l\right)} K_{j, s} d \widetilde{x}\right|=\left|R\left(0, \hat{D}, D_{t}\right) \hat{\Delta}^{(1 / 2)\left(n+s+m_{j}-l\right)} \hat{K}_{j, s}\right| \\
& \quad \leqq \sum_{i \neq j}\left|a_{0 i}(\hat{D}) \hat{\Delta}^{(1 / 2)\left(n+s+m_{j}-l\right)} \hat{B}_{i} \hat{K}_{j, s}\right| \leqq C t\left(|\hat{x}|^{2}+t^{2}\right)^{(1 / 2)(-n+q-1)} .
\end{aligned}
$$

This establishes Theorem 4.2.

CoRollary 4.2. If $\widetilde{D}^{l}$ is any derivative of order $l$ involving at least one differentiation with respect to a component of $\widetilde{x}$, then

$$
\left[\widetilde{D}^{l} w\right]_{\alpha}^{n, t} \leqq C\left[\varphi_{j}\right]_{l-m_{j}+\infty}^{q} .
$$

Proof. The operator $\widetilde{D}^{l}$ is a particular case of the type treated in the theorem but in this case $W_{1}=0$ and $R\left(0, \hat{D}, D_{t}\right)=0$, so that in applying Theorem 2.1 we see that $C_{2}=0$ and the second statement. in that theorem holds.

We shall now return to the system (4.1). Our object will be to find operators $Q(D)$ such that $Q(D) u$ will be estimable in various senses in terms of $f$ and $\varphi_{j}$. Our first result is an immediate consequence of Theorems 3.1 and 4.2. For these we shall think of $t$ as. the $(n+1)$-st component of $x, t=x_{n+1}$, and let $[\tilde{u}]_{l+\infty}^{n+1}$ denote

$$
[\widetilde{u}]_{l+\alpha}^{n+1}=\text { l.u.b. }\left|\hat{D}^{l} u(x)\right|+\text { l.u.b. } \frac{\left|\widetilde{D}^{l} u\left(x_{1}\right)-\widetilde{D}^{l} u\left(x_{2}\right)\right|}{\left|x_{1}-x_{2}\right|^{\alpha}},
$$

where the l.u.b.'s are taken over points $x, x_{1}, x_{2}$ in the domain of definition of $u$, and over derivatives $\widetilde{D}^{l}$ which involve at least one differentiation with respect to a component of $\widetilde{x}$.

THEOREM 4.4. Let the normalized polynomial $P(\xi, \tau)$ of degree $l \geqq 2 m$ satisfy (4.15) and $u, f$, and $\varphi_{j}$ of compact support satisfy (4.1). Then

$$
[P(D) u]_{\alpha}^{q} \leqq C\left([f]_{l-2 m+\alpha}^{n}+\sum_{j}\left[\varphi_{j}\right]_{l-m,+\alpha}^{q}\right) .
$$

Furthermore if $l>\max \left[m_{j}\right]$ and $q>0$, then 


$$
[\tilde{u}]_{l+\alpha}^{n+1} \leqq C\left([f]_{l-2 m+\alpha}^{q}+\sum_{j}\left[\varphi_{j}\right]_{l-m_{j}+\alpha}^{q}\right)
$$

Proof. We use representation (4.2). Theorem 3.1 yields

$$
[\widetilde{v}]_{l+a}^{n+1} \leqq C[f]_{l-2 m+\alpha}^{q},
$$

and

$$
[v]_{l+\alpha}^{n} \leqq C[f]_{l-2 m+\alpha}^{n} .
$$

The latter is obtained directly for derivatives $D^{l} v$ containing at least one differentiation with respect to a component $x_{i}(1 \leqq i \leqq n)$ by setting $q=n$; but we may differentiate $L v=f l-2 m$ times with respect to $x_{n+1}$ and solve for $\partial^{l} v / \partial x_{n+1}^{l}$ in terms of such, thus obtaining the estimate in general.

Thus it follows that

$$
\left[\psi_{j}\right]_{l-m_{j}+\alpha}^{n} \leqq C[f]_{l-2 m+\alpha}^{n}
$$

and

$$
\left[\widetilde{\psi}_{j}\right]_{l-m j^{+\infty}}^{n} \leqq C[f]_{l-2 m+\infty}^{q} \quad \text { (for } l>m_{j} \text { ). }
$$

The former, together with Theorem 4.2, yields (4.18). To derive (4.19) we represent

$$
\widetilde{D}^{\imath} K_{j} * \psi_{j}=D^{m} K_{j} * \widetilde{D}^{\imath-m_{j}} \psi_{j},
$$

then apply Theorem 4.2 with $q=n$ to obtain (4.19).

THEOREM 4.5. All the interior and boundary-type estimates proved so far (i.e., Theorems 3.1 and 4.-4.4) remain true when the smoothness requirements of the functions involved are relaxed to the extent that they have only the differentiability and boundedness properties implied in the statement of the corresponding estimate. For example, (4.18) is true if only $u \in \mathscr{C}_{l+\alpha}^{q}, f \in \mathscr{C}_{l-2 m+\alpha}^{n}$, and $\varphi_{j} \in \mathscr{C}_{l-m,+\alpha}^{q}$.

Proof. The theorem follows from the fact that every function $\varphi \in \mathscr{C}_{l+\infty}^{q}$ may be approximated by functions $\varphi_{\varepsilon} \in \mathscr{C}_{\infty}^{n+1}$ in such a way that $\lim _{\varepsilon \rightarrow 0}\left|\varphi_{\varepsilon}\right|_{l+\alpha}^{q}=|\varphi|_{l+\alpha}^{q}$. The $\varphi_{\varepsilon}$ may, for example, be defined by $\varphi_{\varepsilon}(x)=j_{\varepsilon}(x) * \varphi(x)$, where $j_{\varepsilon}$ is the Friedrichs mollifier, $j_{\varepsilon}(x)=\varepsilon^{-n} j_{1}(x / \varepsilon)$, $j_{1}(x)$ being a function in $\mathscr{C}_{\infty}^{n+1}$ with $\int j_{1}(x) d x=1$, and $j_{1}=0$ for $|x|>1$. Then it is an easy consequence of the "smearing" action of $j_{\varepsilon}$ that $\left|\varphi_{\varepsilon}\right|_{0}^{0} \leqq|\varphi|_{0}^{0}$. Also it is seen that at every point $x$ where $\varphi$ is continuous, $\varphi_{\varepsilon}(x) \rightarrow \varphi(x)$. Since for every $\delta$ we can find such a point of continuity $x$ with $|\varphi(x)|>|\varphi|_{0}^{0}-\delta$, it follows that 
$\lim _{\varepsilon \rightarrow 0} \inf \left|\varphi_{\varepsilon}\right|_{0}^{0} \geqq|\varphi|_{0}^{0}$. Combining the two inequalities, we have $\lim _{\varepsilon \rightarrow 0}\left|\varphi_{\varepsilon}\right|_{0}^{0}=|\varphi|_{0}^{0}$. But the same reasoning may be applied to derivatives and difference quotients of $\varphi$, since these processes commute with the convolution. Hence

$$
\lim _{\varepsilon \rightarrow 0}\left|\varphi_{\varepsilon}\right|_{l+\alpha}^{q}=|\varphi|_{l+\alpha}^{q}
$$

as stated. Now in treating a typical Theorem such as 4.4 , we first continue $u$ a short distance into the region $t \leqq 0$ as a function with the same smoothness properties as it has for $t>0$, then define $u_{\varepsilon}=$ $j_{\varepsilon} * u, f_{\varepsilon}=L u_{\varepsilon}$, and $\varphi_{j \varepsilon}=\left.B_{j} u_{\varepsilon}\right|_{t=0}$. Then the theorem is true for $u_{\varepsilon}$, $f_{\varepsilon}, \varphi_{j \varepsilon} ; \quad$ but $\left[P u_{\varepsilon}\right]_{\alpha}^{q} \rightarrow[P u],\left[f_{\varepsilon}\right]_{l-2 m+\alpha}^{n} \rightarrow[f]_{l-2 m+\alpha}^{n}, \quad$ and $\left[\varphi_{j \varepsilon}\right]_{l m j^{+\alpha}}^{q} \rightarrow$ $\left[\varphi_{j}\right]_{l-m_{j}+\alpha}^{q}$, so it is true as stated.

5. Variable coefficients. The foregoing results concerning equations with constant coefficients in a half-space permit the derivation of certain similar results for more general domains and variable coefficients. The procedure we shall use is basically that in $[2, \S 7]$; however, the arguments here will be more involved, and in the case $q<n$, the results are much less general.

Let $\mathscr{D}$ be a domain in $(n+1)$-dimensional space with boundary $\dot{\mathscr{D}}$, and consider the problem

$$
\begin{aligned}
L(x, D) u & =f(x), & & x \in \mathscr{D}, \\
B_{j}(x, D) u & =\varphi_{j}(x), & & x \in \dot{\mathscr{D}} .
\end{aligned}
$$

$L(x, D)$ is assumed to be uniformly elliptic in $\mathscr{D}$ with ellipticity constant $E$, and to satisfy the root condition of [2]. Also the $B_{j}$ are to satisfy the complementing condition of [2] with "determinant constant" 4 .

As before let $q$ be an integer, $0 \leqq q \leqq n$, and $l$ an integer with $l \geqq \max \left[2 m, m_{j}\right]$; but now we permit the $=$ sign in this latter inequality to hold only in the case $m_{j}<2 m$ for all $j$. Let $\mu_{0}=\max$ $[1, l-2 m]$ and $\mu_{j}=\max \left[1, l-m_{j}\right]$. We assume the coefficients of $L$ and $B_{j}$ to belong to classes $\mathscr{C}_{\mu_{0}+\alpha}^{n+1}(\mathscr{D})$ and $\mathscr{C}_{\mu_{j}+\alpha}^{n+1}(\dot{\mathscr{D}})$ respectively, and to have $|\cdot|_{\mu_{0}+\alpha}^{n+1}$ and $|\cdot|_{\mu_{j+\alpha}}^{n+1}$ norms bounded by the constant $H$.

In addition to these assumptions on $L$ and $B_{j}$, we shall require that coordinate tranformations may be introduced which, at least locally, flatten out the boundary $\dot{\mathscr{D}}$, and such that the operators $L$ and $B_{j}$ transform into operators of a special type. This special type is that in which the coefficients of all derivatives of order $2 m$ in $L$ and those of order $m_{j}$ in $B_{j}$, which involve only differentiations with respect to "undistinguished" variables, be constant on the new flat boundary. As will be shown in $\S 6$, this assumption involves no loss 
of generality when $q=n$ (this is the case when there is one "undistinguished" direction, and it is normal to $\dot{\mathscr{D}}$ ). However for $q<n$ it limits substantially the generality of the results. There is one exception however: the case when $m=1, B_{1} \equiv 1, q=0$, and $n=1$ or 2. In this case such transformations as required above are always possible; however in this case the same a priori estimates may be obtained much more easily by use of the known maximum principle for second order elliptic equations.

Theorem 5.1 treats the case when the domain $\mathscr{D}$ is the halfspace $x_{n+1}>0$, and $L$ and $B_{j}$ are of the special type. Theorem 5.4 indicates the same results to hold if $L$ and $B_{j}$ may be transformed locally to operators of the special type, $\dot{\mathscr{D}}$ at the same time being flattened locally. Theorem 5.2 treats the case when $L$ and $B_{j}$ are of special type throughout $\mathscr{D}$; then the full Hölder continuity of $f$ is no longer required.

Constants appearing in this and the following section which depend only on $E, \Delta, \mathrm{H}, m, m_{j}, \alpha$, and $l$ will all be denoted by the letter $C$. Whenever an operator appears with a tilde $(\sim)$ over it, it is to be understood that every term of the operator involves at least one differentiation with respect to a component of $\tilde{x}$ or in a "distinguished direction.". Symbols such as $|\cdot| \stackrel{q}{\mathscr{D}_{1}}$, where $\mathscr{D}_{1}$ is a subdomain of $\mathscr{D}$, simply mean the same as $|\cdot|^{q} \ldots$, except that the function in brackets is considered to have only $\mathscr{D}_{1}$ as its domain of definition. We shall also use the symbol $|\widetilde{u}|_{l+\infty}^{n+1}$ as defined on page 526 . An operator $Q(x, D)$ with variable coefficients is said to be normalized if the l.u.b. of all its coefficients for all $x$ in its domain of definition is one.

Theorem 5.1. Let $L(x, D)$ and $B_{j}(x, D)$ satisfy the above conditions, and in addition assume $L$ and $B_{j}$ to be of the forms

$$
\begin{aligned}
& L(x, D)=L_{0}(D)+\widetilde{L}(x, D)+L_{1}(x, D)+\text { lower order terms } \\
& B_{j}(x, D)=B_{j_{0}}(D)+\widetilde{B}_{j}(x, D)+\text { lower order terms }{ }^{1}
\end{aligned}
$$

where $L_{0}$ and $B_{j_{0}}$ have constant coefficients, and $L_{1}$ has coefficients which vanish for $x_{n+1}=0$. Let $u(x), f(x)$, and $\varphi_{j}(x)$ satisfy (5.1) in the half-space $x_{n+1}>0$, and have smoothness and boundedness properties which will guarantee the norms in (5.5) and in the proof of the theorem to exist. Let $P(x, D)$ be any homogeneous normalized operator of degree $l$ with coefficients in $\mathscr{C}_{l+\infty}^{n+1}$ whose $|\cdot|_{l+\infty}^{n+1}$ norms are

1 Terms of the form $R(x, D) B_{i}(x, 0)(i \neq j), R$ an operator of degree $m_{j}-m_{i}$, would also be permissible in the expression for $B_{j}$; but if they are present we may replace $B_{j}$ by $B_{j}-R B_{i}$ and $\varphi_{j}$ by $\varphi_{j}-R(x, D) \varphi_{i}$, obtaining an equivalent boundary-value problem in which they no longer appear. 
bounded by $H$, and which may be represented in the form

$$
P(x, D)=A(x, D) L_{0}(D)+\sum_{j} a_{j}(x, D) B_{j 0}(D)+\widetilde{P}(x, D)+P_{1}(x, D) .
$$

where $P_{1}$ vanishes for $x_{n+1}=0$, ond the $a_{j}$ involve no differentiations with respect to $x_{n+1}$. Then

$$
\begin{aligned}
& |P(x, D) u|_{\alpha}^{q} \leqq C\left\{|f|_{l-2 m+\alpha}^{n+1}+\sum_{j=1}^{m}\left|\varphi_{j}\right|_{l-m,+\alpha}^{q}+|u|_{0}^{0}\right\}, \\
& |\tilde{u}|_{l+\alpha}^{n+\alpha} \leqq C\left\{|f|_{l-2 m+\infty}^{n+1}+\sum_{j=1}^{m}\left|\varphi_{j}\right|_{l-m}^{q} g_{+\infty}^{+\infty}+|u|_{0}^{0}\right\} .
\end{aligned}
$$

Proof. The proof will employ the following two lemmas, the first of which is contained in the results of [2].

Lemma 5.1. Let $u \in \mathscr{C}_{l-1+\alpha}^{n+1}, f \in \mathscr{C}_{l-2 m+\alpha}^{n+1}, \varphi_{j} \in \mathscr{C}_{l-m_{j}}^{0}$ be solutions to (5.1) in an arbitrary domain $\mathscr{D}$ with smooth enough boundary. Then

$$
|u|_{l-1+\alpha}^{n+1} \leqq C\left\{|f|_{l-2 m+\alpha}^{n+1}+\sum_{j}\left|\varphi_{j}\right|_{l-m_{j}}^{0}+|u|_{0}^{0}\right\} \text {. }
$$

Proof. If $l>\max \left[2 m, m_{j}\right]$ this follows directly from [2, Theorem 7.3]: there $\mathscr{A}$ is identified with $\mathscr{D}, l$ is replaced by $l-1$, and the inequalities $|f|_{l-2 m-1+\alpha}^{n+1} \leqq|f|_{l-2 m-1+\alpha}^{n+1}$ and $\left|\varphi_{j}\right|_{l-m_{j-1+\alpha}}^{n+1} \dot{\mathscr{D}} \leqq\left|\varphi_{j}\right|_{l-m}^{0} \dot{\mathscr{D}}$, are employed. The other possibility is that $\max \left[m_{j}\right]<2 m$ and $l=2 m$. Let. $S_{1}$ and $S_{2}$ be concentric balls with radii 1 and 2 respectively, and center in $\mathscr{D}$. Let $a$ be some number such that the hyperplane $x_{1}=a$ intersects $S_{2}$. Define $F_{1}(x)=\int_{a}^{x_{1}} f\left(\xi, x_{2}, \cdots, x_{n+1}\right) d \xi$ (we may need to extend $f$ outside $\mathscr{D}$ for this to be defined), and $F_{\beta}=0$ for $\beta>1$. Then Theorem 9.3 of [2] is applicable: set $p=2 m-1$ and $\mathscr{A}=S_{1}$. The conclusion of that theorem is:

$$
|u|_{l-1+\alpha}^{n+1} S_{1} \leqq C\left\{\sum_{\beta}\left|F_{\beta}\right|_{\alpha}^{n+1, S_{2}}+\sum_{j}\left|\varphi_{j}\right|_{2 m-1-m_{j}+\alpha}^{n+1}+|u|_{0}^{0}\right\} .
$$

But $\left|F_{1}\right|_{\alpha}^{n+1, S_{2}} \leqq 4|f|_{\alpha}^{n+1}$ and $F_{\beta}=0, \beta>1$, so (5.6) holds in this case also if a superscript $S_{1}$ is adjoined to the norm on the left. But it. does not appear on the right and its center is arbitrary, so (5.6) is valid as written.

Lemma 5.2. Consider again the case when the domain $\mathscr{D}$ is the halfspace $x_{n+1}>0$. Let $b(x)$ be a function in $\mathscr{C}_{l+\infty}^{n+1}$ such that $b(x)=0$ for $x_{n+1}=0$, and $|b|_{l+\infty}^{n+1}<H$. Then for every derivative of order $l$,

$$
\left|b(x) D^{l} u\right|_{\alpha}^{n+1} \leqq C\left\{|f|_{l-2 m+\alpha}^{n+1}+\sum_{j}\left|\varphi_{j}\right|_{l-m,}^{0}+|u|_{0}^{0}\right\}
$$


Proof. Let $v-b(x) u$. Then

$$
\begin{aligned}
& L(x, D) v=b f+\sum_{0 \leqq k \leqq 2 m-1} c_{k}(x) D^{k} u=F(x), \\
& \left.B_{j} v\right|_{x_{n+1}=0}=\left.\sum_{k<m_{j}} e_{j_{k}}(x) D^{k} u\right|_{x_{n+1}=0}=\Phi_{j}(x) .
\end{aligned}
$$

It follows from Lemma 5.1 that

$$
\left.|F|\right|_{l-2 m+\infty} ^{n+1} \leqq C\left\{|f|_{l-2 m+\infty}^{n+1}+\sum_{j}\left|\varphi_{j}\right|_{l-m,}^{0}+|u|_{0}^{0}\right\}
$$

and

$$
\left|\Phi_{j}\right|_{l-m_{j}+\alpha}^{n} \leqq C\left\{|f|_{l-2 m}^{n+1}+\sum_{j}\left|\varphi_{j}\right|_{l-m}^{0}+|u|_{0}^{0}\right\} .
$$

Hence from the main boundary estimate of [2] (Theorem 7.3),

$$
|v|_{l+\alpha}^{n+1} \leqq C\left\{|f|_{l-2 m+\infty}^{n+1}+\sum_{j}|\varphi|_{l-m,}^{0}+|u|_{0}^{0}+|v|_{0}^{0}\right\} \text {. }
$$

But since for every derivative $D^{l}$ we have

$$
b D^{\imath} u=D^{l} v+\text { lower order terms }
$$

and since the lower order terms may be estimated by (5.6), and also since $|v|_{0}^{0} \leqq C|u|_{0}^{0}$, (5.7) follows.

Now to proceed with the proof of Theorem 5.1, let $\mathscr{E}_{0}$ be the class of homogeneous operators of degree $l$ which have a representation of the form (5.4), and whose coefficients

(1) are in $\mathscr{C}_{a}^{n+1}$ and have $|\cdot|_{a}^{n+1}$ norms bounded by $H$; and

(2) have first derivatives with respect to $x_{n+1}$ in $\mathscr{C}_{a}^{n+1}$ with norms $|\cdot|_{a}^{n+1}$ bounded by $H$. Let $\mathscr{E}$ be the subclass consisting of those operators in $\mathscr{E}_{0}$ with coefficients in $\mathscr{C}_{1+\infty}^{n+1}$ whose $|\cdot|_{1+\infty}^{n+1}$ norms are bounded by $H$. Let $\delta$ be a fixed number, $0<\delta<1$, which will be defined later. We define the number $M$ as

$$
M=4 \delta^{-\alpha} \operatorname{lub}_{\mathscr{E}_{0}}|\bar{Q}(x, D) u|_{0}^{0}+\operatorname{lub}_{\mathscr{E}}|\bar{Q}(x, D) u|_{\alpha}^{q}+[\widetilde{u}]_{l+\alpha}^{n+1}
$$

with the lub's taken over all operators $\bar{Q}(x, D) \in \mathscr{E}_{0}$ and $\mathscr{E}$ respectively. Then from the definition of $|\cdot|_{\alpha}^{q}$ there is a point $y$ and an operator $Q(x, D) \in \mathscr{E}_{0}$ or in $\mathscr{E}$, or a derivative $\widetilde{D}^{l}$, such that one of the following four quantities is $>\frac{1}{8} M$ :

$$
\begin{aligned}
U_{1} & =4 \delta^{-\alpha}|Q(y, D) u(y)|, \\
U_{2} & \left.=\frac{|Q(z, D) u(z)-Q(y, D) u(y)|}{|\widetilde{z}-\widetilde{y}|^{\alpha}} \text { (for some } z \text { with } \hat{z}=\hat{y}\right), \\
U_{3} & =\left|\widetilde{D}^{l} u(y)\right|, \\
U_{4} & =\frac{\left|\widetilde{D}^{l} u(z)-\widetilde{D}^{l} u(y)\right|}{|z-y|^{\alpha}} \quad \text { (for some } z \text { ). }
\end{aligned}
$$


with $U_{2}$ and $U_{4}$ missing if $q=0$. We shall carry out the proof first under the assumption that $U_{2}>\frac{1}{8} M$. The proof for other cases will then require only slight additional arguments. Therefore we assume $q>0, Q \in \mathscr{E}$, and $U_{2}>\frac{1}{8} M$. It may be assumed that $|z-y| \leqq \delta$ since if not, the quotient $U_{2}$ will be $<2 \delta^{-\alpha}|Q u|_{0}^{0}$, and there will be a point $y^{\prime}$ such that

$$
U_{1}^{\prime}=4 \delta^{-\alpha}\left|Q\left(y^{\prime}, D\right) u\left(y^{\prime}\right)\right|>2 \delta^{-\alpha}|Q u|_{0}^{0}>U_{2}>\frac{1}{8} M .
$$

The argument thus reduces to the case when the first of the four quantities in (5.8) is $>\frac{1}{8} M$, which case is treated separately.

Let $\zeta(t)$ be a $\mathscr{C}_{\infty}$ function of a single variable such that $\zeta(t)=1$ for $|t|<1$ and $\zeta(t)=0$ for $|t|>2$. Define

$$
w(x)=\zeta\left(\frac{x-y}{\delta}\right) u(x)
$$

if both $y$ any $z$ are further than $2 \delta$ from $\dot{\mathscr{D}}$; i.e., both $y$ and $z$ have $(n+1)$-st component $\geqq 2 \delta$; and

$$
w(x)=\zeta\left(\frac{x-y_{B}}{3 \delta}\right) u(x)
$$

if either $y$ or $z$ is nearer than $2 \delta$ from $\dot{\mathscr{D}}$; here $y_{B}$ is the projection of $y$ onto $\dot{\mathscr{D}}$, so that if $y=\left(y_{1} \cdots y_{n+1}\right), y_{B}=\left(y_{1}, \cdots, y_{n}, 0\right)$.

Let us assume the latter alternative $(5.9 \mathrm{~b})$ to be the case; the proof for the former is similar. First, on the basis of (5.4), also considering (5.2) and (5.3), we may express $Q(y, D)$ as

$$
Q(y, D)=Q_{1}(y, D)+Q_{2}(y, D),
$$

where $Q_{2}$ vanishes for $y$ on $\dot{\mathscr{D}}$ and

$$
Q_{1}(y, D)=A(y, D) L^{\prime}\left(y_{B}, D\right)+\sum_{j} a_{j}(y, D) B_{j}^{\prime}\left(y_{B}, D\right)+\widetilde{Q}_{3}(y, D),
$$

where $L^{\prime}$ and $B_{j}^{\prime}$ are those parts of $L$ and $B_{j}$ consisting of highest order terms only. Let us decompose the quotient $U_{2}$ as follows:

$$
\frac{1}{8} M<\frac{|Q(z, D) u(z)-Q(y, D) u(y)|}{|\widetilde{z}-\widetilde{y}|} \leqq T_{1}+T_{2}+T_{3},
$$

where

$$
\begin{aligned}
& T_{1}=\left|\frac{Q(z, D)-Q(y, D)}{|\widetilde{z}-\widetilde{y}|^{\alpha}} u(z)\right|, \\
& T_{2}=\frac{Q_{1}(y, D) u(z)-Q_{1}(y, D) u(y) \mid}{|\widetilde{z}-\widetilde{y}|^{\alpha}},
\end{aligned}
$$




$$
T_{3}=\frac{\left|Q_{2}(y, D) u(z)-Q_{2}(y, D) u(y)\right|}{|\widetilde{z}-\widetilde{y}|^{\alpha}} .
$$

Owing to the smoothness of the coefficients of $Q$ and to the definiton of $\mathscr{E}_{0}$, we have

$$
T_{1} \leqq C \delta^{1-\alpha} \operatorname{lub}_{\bar{Q} \in \mathscr{E}}|\bar{Q}(x, D) u(x)|_{0}^{0} \leqq C \delta M .
$$

Theorem 4.4 (4.18), with Theorem 4.5 may be invoked to estimate $T_{2}$. In view of the definition of $Q_{1}$, that theorem tells us the following (where we have used $u(y)=w(y), u(z)=w(z)$; notice also that condition (4.15) "neutralizes" $\widetilde{Q}_{3}$ ):

$$
\begin{aligned}
T_{2} & =\frac{\left|Q_{1}(y, D) w(z)-Q_{1}(y, D) w(y)\right|}{|\widetilde{z}-\widetilde{y}|} \\
& \leqq C\left\{\left[L^{\prime}\left(y_{B}, D\right) w(x)\right]_{l-2 m+\alpha}^{n}+\sum_{j}\left[B_{j}^{\prime}\left(y_{B}, D\right) w\right]_{l-m,+\alpha}^{q}\right\}
\end{aligned}
$$

$\left(y_{B}\right.$, of course, is to be considered a constant when the norms on the right are computed.) To further estimate the terms on the right, we introduce the symbol $S_{\delta}$ to denote the sphere of radius $6 \delta$ about $y_{B}$. First, for any derivative $D^{\imath-2 m}$,

$$
D^{l-2 m} L^{\prime}\left\{\left(y_{B}, D\right) w=\zeta D^{l-2 m} L^{\prime}\left(y_{B}, D\right) u+\sum_{k \geq 1} \gamma_{k}(x)\left(D^{k} \zeta\right) D^{l-k} u\right.
$$

From (5.1), (5.2),

$$
\begin{aligned}
D_{x}^{l-2 m} L^{\prime}\left(y_{B}, D\right) u(x)= & D^{l-2 m} L(x, D) u(x)+D_{x}^{l-2 m}\left(\widetilde{L}\left(y_{B}, D\right)\right. \\
& -\widetilde{L}(x, D)) u(x)-D^{l-2 m} L_{1}(x, D) u(x)+\text { l.o.t. } \\
= & D^{l-2 m} f(x)+Q^{*}(x, D) u-L_{1}(x, D) D^{l-2 m} u(x) \\
& + \text { l.o.t. }
\end{aligned}
$$

where

$$
Q^{*}(x, D)=\left(\widetilde{L}\left(y_{B}, D\right)-\widetilde{L}(x, D)\right) D^{\imath-2 m}
$$

Also

$$
\left[\zeta D^{l-2 m} f(x)\right]_{x}^{n} \leqq[f]_{l-2 m+\alpha}^{n}+\delta^{-\alpha}[f]_{l-2 u}^{0} \leqq C(\delta)[f]_{l-2 m+\alpha}^{n} .
$$

Now since the coefficients of $\widetilde{L}$ are in $\mathscr{C}_{1+\alpha}^{n+1}, Q^{*}$ is a combination of derivatives of the form $\widetilde{D}^{l}$ with coefficients bounded in $|\cdot|_{\alpha}^{n+1}$ norm by $6 H \delta$, for $x \in S_{\delta}$. Since also $|\zeta|_{\alpha}^{n+1}<C \delta^{-\alpha}$, we have

$$
\left[\zeta Q^{*}(x, D) u\right]_{\alpha}^{n} \leqq C\left(\delta[\tilde{u}]_{l+\alpha}^{n}+\delta^{1-\alpha}[\tilde{u}]_{l}^{?}\right) .
$$

By a standard calculus lemma (see for example $[2, \S 5]$ ),

$$
[\tilde{u}]_{l}^{0}<\varepsilon[\tilde{u}]_{l+\alpha}^{n}+C(\varepsilon)|u|_{0}^{0}
$$


for arbitrarily small $\varepsilon$. Choosing $\varepsilon=\delta^{\alpha}$, we have

$$
\left[\zeta Q^{*} u\right]_{\alpha}^{n} \leqq C \delta M+C(\delta)|u|_{0}^{0} .
$$

Lemma 5.1 easily yields

$$
\left[\zeta \cdot(\text { lower order terms) }]_{a}^{n} \leqq C\left\{\left.f\right|_{l-2 m} ^{0}+\Sigma\left|\varphi_{j}\right|_{l-m}^{0}+|u|_{0}^{0}\right\} .\right.
$$

For the same reasons

$$
\left[\gamma_{k}(x)\left(D^{k} \zeta\right)\left(D^{l-k} u\right)\right]_{\alpha}^{n} \leqq C(\delta)\left\{|f|_{l-m_{j}+\alpha}^{0} \sum_{j}\left|\varphi_{j}\right|_{l-2 m+\infty}^{0}+|u|_{0}^{0}\right\}
$$

Combining this result with (5.14-18), we find

$$
\begin{gathered}
{\left[L^{\prime}\left(y_{B}, D\right) w\right]_{l-2 m+\alpha}^{n} \leqq C \delta M+C(\delta)\left\{|f|_{l-2 m+\infty}^{n}+\Sigma\left|\varphi_{j}\right|_{l-m_{j}}^{0}+|u|_{0}^{0}\right\}} \\
+\left[\zeta L_{1}(x, D) D^{l-2 m} u\right]_{\alpha}^{n} .
\end{gathered}
$$

In exactly the same way one obtains

$$
\left[B_{j}^{\prime}\left(y_{B}, D\right) w\right]_{l-m_{j}+\alpha}^{q} \leqq C \delta M+C(\delta)\left\{|f|_{l-2 m}^{0}+\Sigma\left|\varphi_{j}\right|_{l-m_{j}+\alpha}^{q}+|u|_{0}^{0}\right\} .
$$

Combining these results with (5.13), (5.12), and (5.11), we have

$$
\begin{aligned}
M \leqq C_{2} \delta M & +C(\delta)\left\{|f|_{l-2 m+\alpha}^{n}+\Sigma\left|\varphi_{j}\right|_{l-m_{j}+\alpha}^{q}+|u|_{0}^{0}\right\} \\
& +\left[\zeta L_{1}(x, D) D^{l-2 m} u\right]_{\alpha}^{n}+T_{3},
\end{aligned}
$$

with $C_{2}$ independent of $\delta$. The last two terms may be estimated with the use of Lemma 5.2. We shall illustrate the method by estimating $T_{3}$. By hypothesis the coefficients of $Q_{2}$ are in $\mathscr{C}_{1+\alpha}^{n+1}$ and vanish for $x_{n+1}=0$; hence we may take out a factor $x_{n+1}$ from each and have left a function in $\mathscr{C}_{a}^{n+1}$. More specifically, define $b\left(x_{n+1}\right)$ to be an infinitely differentiable function with $|b|_{l+a}^{n+1}<H$ assuming the values

$$
b\left(x_{n+1}\right)= \begin{cases}x_{n+1} & \left(0 \leqq x_{n+1} \leqq 1 / 2\right) \\ 1 & \left(1 \leqq x_{n+1}\right)\end{cases}
$$

Then $b$ may be factored out, and we have

$$
Q_{2}(x, D)=b\left(x_{n+1}\right) Q_{4}(x, D)
$$

where the coefficients of $Q_{4}$ are in $\mathscr{C}_{a}^{n+1}$ with $|\cdot|_{a}^{n+1}$ norms bounded by $H$. Since $b$ satisfies the hypotheses of Lemma 5.2,

$$
\left|b\left(x_{n+1}\right) Q_{4}(y, D) u(x)\right|_{a}^{n+1} \leqq C\left\{|f|_{l-2 m+\alpha}^{n+1}+\sum_{j}\left|\varphi_{j}\right|_{l-m_{j}}^{0}+|u|_{0}^{0}\right\} \text {. }
$$

But since $z_{n+1}=y_{n+1}$, we may write

$$
T_{3}=\frac{\left|b\left(y_{n+1}\right) Q_{4}(y, D) u(z)-b\left(y_{n+1}\right) Q_{4}(y, D) u(y)\right|}{|\widetilde{z}-\widetilde{y}|^{\alpha}}
$$




$$
\begin{aligned}
& =\frac{\left|b\left(z_{n+1}\right) Q_{4}(y, D) u(z)-b\left(y_{n+1}\right) Q_{4}(y, D) u(y)\right|}{|\widetilde{z}-\widetilde{y}|^{\alpha}} \\
& \leqq\left|b\left(x_{n+1}\right) Q_{4}(y, D) u(x)\right|_{\alpha}^{n+1} \leqq C\left\{|f|_{l-2 m+\alpha}^{n+1}+\Sigma\left|\varphi_{j}\right|_{l-m_{j}}^{0}+|u|_{0}^{0}\right] .
\end{aligned}
$$

The same estimate holds for the next to last term in (5.19), so that in all,

$$
M \leqq C_{2} \delta M+C(\delta)\left\{|f|_{l-2 m+\alpha}^{n+1}+\Sigma\left|\varphi_{j}\right|_{l-m_{j}+\alpha}^{q}+|u|_{0}^{0}\right\} .
$$

If any one of the other three quantities $U_{1}, U_{3}$, or $U_{4}$ in (5.8) is assumed to be $>\frac{1}{8} M$, then an inequality similar to (5.21) with other constants $C_{1}, C_{3}, C_{4}$, all independent of $\delta$, may be derived, In the case $U_{1}>\frac{1}{8} M$, then we define $w$ again according to (5.9) (forgetting about $z$ ). $\quad T_{1}$ will be missing from (5.11) and $T_{2}$ and $T_{3}$ are no longer quotients, but rather $4 \delta^{-\alpha}\left|Q_{1}(y, D) u(y)\right|$ and $4 \delta^{-\alpha}\left|Q_{2}(y, D) u(y)\right|$ respectively. Theorem 4.5 again yields (5.13) except for an extra factor $\delta^{-\alpha}$ on the right. Repeating the argument from this point on, we obtain (5.21) with $C_{2} \delta M$ replaced by $C_{1} \delta^{1-\alpha} M$. $U_{3}$ and $U_{4}$ may be treated in similar manners.

Now the definition of $\delta$ is clear:

$$
\delta=\min \left[\left(2 C_{1}\right)^{-1 / 1-\alpha},\left(2 C_{2}\right)^{-1},\left(2 C_{3}\right)^{-1},\left(2 C_{4}\right)^{-1}\right],
$$

so that $C_{1} \delta^{1-\alpha} \leqq 1 / 2$, and $C_{i} \delta \leqq 1 / 2(i=2,3,4)$. Putting all terms in $M$ on the left, (5.21) now implies (5.5a). Also since $|\tilde{u}|_{l+\alpha}^{n+1}=|\tilde{u}|_{l-1+\alpha}^{n+1}+$ $[\tilde{u}]_{l+\infty}^{n+1} \leqq M+|\widetilde{u}|_{l-1+\infty}^{n+1}$ and since the last term here may be estimated with Lemma 5.1, (5.5b) is deduced and the theorem proved.

The condition (5.2) imposed on $L$ is really only a condition on $L$ at the boundary $x_{n+1}$; consequently the full Hölder-continuity of $f$ is needed for the estimate (5.5). The following theorem will only utilize $f$ 's Hölder continuity with respect to $\tilde{x}$; but as a price for it a condition on $L$ analogous to (5.2) is imposed throughout the domain; and also the class of operators which are estimable is reduced.

THEOREM 5.2. Let $L, B_{j}, u, f$, and $\varphi_{j}$ satisfy the hypotheses of Theorem 5.1, except that $f$ is required merely to be in $\mathscr{C}_{l-2 m+\alpha}^{q}$, and $L(x, D)$ is of the form

$$
L(x, D)=L_{0}(D)+\widetilde{L}(x, D)+\text { lower order terms } .
$$

Then

$$
|\tilde{u}|_{l+\alpha}^{n+1} \leqq C\left\{|f|_{l-2 m+\alpha}^{q}+\sum\left|\varphi_{j}\right|_{l-m_{j}+\alpha}^{q}+|u|_{0}^{0}\right\} .
$$

Proof. The proof is the same in outline as that of Theorem 5.1; the following are the only differences. The pseudonorm $[\widetilde{u}]_{l+\infty}^{n+1}$ takes the place of $M$, so that $U_{1}$ and $U_{2}$ are missing from the list in (5.8). 
Taking the case $U_{4} \geqq 1 / 8[\tilde{u}]_{l+\alpha}^{n+1}$, Theorem 4.4 (4.18) is again invoked to yield (5.22). Again this takes the place of (5.13) and the argument is the same, except that the superscripts $n$ in (5.16-19) are to be replaced by $q$, and last two terms in (5.19) are now missing. This proves the theorem.

Theorems 5.1 and 5.2 were based on the assumption that $\mathscr{D}$ is, or may be mapped onto, a half-space in such a manner that the transformed operators $L$ and $B_{j}$ satisfy certain properties. The following theorem serves to indicate that such a transformation property of $\mathscr{D}, L$, and $B_{j}$ need only be local; i.e., we assume only that every point in $\mathscr{D}$ near the boundary has a neighborhood which may be mapped onto a hemisphere, $L$ and $B_{j}$ being transformed under this mapping in the desired manner.

Specifically, we assume that some portion $\Gamma_{1}$ of the boundary $\dot{\mathscr{D}}$ (it may happen that $\Gamma_{1}=\dot{\mathscr{D}}$ ) is covered by a network of $q$ families of "distinguished curves," each of class $\mathscr{S}_{l+\alpha}^{n+1}$, with no two curves of the same family intersecting each other, and no two curves from any two families tangent at any point. Then there will be $q$ curves, one from each family, passing through each point in $\Gamma_{1}$. It is along these curves that we shall assume certain functions to be Hölder continuous. If $\Gamma_{1} \neq \dot{\mathscr{D}}$, we speak of another portion $\Gamma_{2}$ with $\Gamma_{1} \cup \Gamma_{2}=$ $\dot{\mathscr{D}}$, and $\Gamma_{2}$ overlapping $\Gamma_{1}$ so that the boundaries $\Gamma_{1}$ and $\Gamma_{2}$ are bounded away from each other by some number $d_{1}>0$. We also assume these $q$ families may be extended in some manner to cover a subdomain $\mathscr{D}_{1}$ adjacent to $\Gamma_{1}, \mathscr{D}_{1}$ having the properties that $\dot{\mathscr{D}}_{1} \cap$ $\dot{\mathscr{D}}=\Gamma_{1}$, every point of $\mathscr{D}_{1}$ is nearer than $2 d_{1}$ to $\Gamma_{1}$, and every point of $\mathscr{D}-\mathscr{D}_{1}$ is further than $d_{1}$ from $\Gamma_{1}-\Gamma_{2}$.

Our smoothness assumptions on $\dot{\mathscr{D}}$ will be very much the same as those made in [2, Theorem 7.3]. First of all, we assume $\Gamma_{2}$ to be of class $\mathscr{C}_{l+a}^{n+1}$ and to satisfy the other requirements which are imposed in Theorem 7.3 of [2] on the boundary portion $\Gamma$ spoken of there. Next, concerning $\Gamma_{1}$ and $\mathscr{D}_{1}$, we suppose there is some number $d \leqq d_{1}$ such that evey point $y \in \mathscr{D}_{1}$ has a neighborhood $N_{y}$ whose boundary contains a portion of $\Gamma_{1}$ and which may be mapped by a one-to-one $\mathscr{C}_{l+\infty}^{n+1}$ mapping $\mathscr{T}_{y}$ onto the hemisphere $\mathscr{H}\left(|\bar{x}|=1, \bar{x}_{n+1}\right.$ $>0)$ of radius 1 and center at origin in $(n+1)$-dimensional $\bar{x}$-space in such a manner that the following conditions are fulfilled:

(1) The image of $y$ is closer than $1 / 3$ to the origin.

(2) $\dot{N}_{y} \cap \dot{\mathscr{D}}$ is mapped onto the flat portion $\dot{\mathscr{H}}_{0}$ of the boundary of $\mathscr{H}$. Also, denoting the image of $N_{y} \cap \mathscr{D}_{1}$ by $\mathscr{H}_{1 y}$, the distinguished curves in $N_{y} \cap \mathscr{D}_{1}$ are to be mapped onto line segments in $\mathscr{H}_{1 y}$ which are parallel to the first $q$ coordinate axes. In accordance with our usual practice, the first $q$ coordinates of a point $\bar{x}$ in $\mathscr{H}_{1 y}$ 
will be grouped together in $\tilde{\bar{x}}$, and the others in $\hat{\bar{x}}$ (these will not be defined outside $\mathscr{K}_{1 y}$ ).

(3) $L$ and $B_{j}$ are transformed into new operators $L_{y}$ and $B_{j y}$ with the same smoothness, ellipticity, and complementing conditions as the original ones. We assume the same constant $H$ will serve for the transformed operators independently of $y$.

(4) The transformed operators $L_{y}$ and $B_{j y}$ may be expressed, in $\mathscr{H}$, in the form:

$$
\begin{aligned}
L_{y}(\bar{x}, \bar{D})=\lambda_{y}(\bar{x}) L_{0 y}(\bar{D}) & +\widetilde{L}_{y}(\bar{x}, \bar{D})+L_{1 y}(\bar{x}, \bar{D}) \\
& + \text { lower order terms },
\end{aligned}
$$

where $\bar{D}$ denotes differentiation with respect to the $\bar{x}, L_{0 y}$ is an operator with constant coefficients, $L_{1 y}$ vanishes on $\dot{\mathscr{C}}_{0}$ (i.e., for $\bar{x}_{n+1}=0$ ); and for $\bar{x} \in \mathscr{H}_{1 y}$, each term of $\widetilde{L}_{y}$ involves a differentiation with respect to a component of $\overline{\bar{x}}$ (this with be true of all operators below with a “ "). Also for $\bar{x}_{n+1}=0$,

$$
B_{j y}(\bar{x}, \bar{D})=\beta_{y}(\bar{x}) B_{j 0 y}(\bar{D})+\widetilde{B}_{j y}(x, \bar{D})+\text { l.o.t. }
$$

Note that the ellipticity and complementing conditions guarantee $\lambda_{y}$ and $\beta_{y}$ to be bounded away from zero by a constant depending on $H$.

Referring back to the original coordinate system, let $\mathscr{E}$ be the class of operators $P(x, D)$ defined in $\mathscr{D}$ with coefficients in $\mathscr{C}_{1+\alpha}^{n+1}$, whose $|\cdot|_{1+\infty}^{n+1}$ norms are bounded by $H$, with the property that when subjected to any transformation $\mathscr{T}_{y}, P$ assumes the form

$$
P_{y}(\bar{x}, \bar{D})=A_{y}(\bar{x}, \bar{D}) L_{0 y}(\bar{D})+\Sigma a_{j}(\bar{x}, \bar{D}) B_{j 0 y}(\bar{D})+\widetilde{P}_{y}(\bar{x}, \bar{D})+P_{1 y}(\bar{x}, \bar{D}),
$$

where $\widetilde{P}_{y}$ and $P_{1 y}$ have the same properties as $\widetilde{L}_{y}$ and $L_{1 y}$. Let $\tilde{\mathscr{E}}$ be the subset of $\mathscr{E}$ consisting of those $P$ which assume the form

$$
P_{y}(\bar{x}, \bar{D})=\widetilde{P}_{y}(\bar{x}, \bar{D})
$$

with each transformation $\mathscr{T}_{y}$. Of course for points $x$ in $\mathscr{D}-\mathscr{D}_{1}$ there are no distinguished directions and consequently there is no condition (except smoothness) imposed on $P(x, D)$ there.

The symbol $[\psi(x)]_{l+\alpha}^{a \mathscr{D}_{1}}$ will be used below to denote

$$
[\psi]_{l+\infty}^{q} \mathscr{D}_{1}=\operatorname{lub}\left|D^{l} \psi(x)\right|+\operatorname{lub} \frac{\left|D^{l} \psi\left(x_{1}\right)-D^{l} \psi\left(x_{2}\right)\right|}{\left|x_{1}-x_{2}\right|^{\alpha}},
$$

where the first lub is over all points $x \in \mathscr{D}_{1}$ and derivatives of order $l$; the second, over all derivatives $D^{\imath}$ and points $x_{1}, x_{2} \in \mathscr{D}_{1}$ such that whenever $x_{2} \in N_{x_{1}}$, the images $\bar{x}_{1}$ and $\bar{x}_{2}$ have the same components $\hat{\bar{x}}$ (i.e., $x_{1}$ and $x_{2}$ may be joined by a curve pieced together from portions of distinguished curves). A similar meaning is attached to $|\psi|_{l+\infty}^{q, Z_{1}}$ 
and $|\psi|_{l+a}^{q} r_{1}$.

THEOREM 5.3. Let $L, B_{j}$, and $\mathscr{D}$ satisfy the above conditions. Let $u, f$, and $\varphi_{j}$ satisfy (5.1) and have the differentiability properties implied below. Then if $P(x, D) \in \mathscr{E}$,

$$
\begin{gathered}
|P(x, D) u|_{a}^{q, \mathscr{D}_{1}} \leqq C\left\{|f|_{l-2 m+\alpha}^{n+1}+\Sigma\left|\varphi_{j}\right|_{l-m_{j}+\alpha}^{q \Gamma_{1}-\Gamma_{2}}+\Sigma\left|\varphi_{j}\right|_{l-m_{j}+\alpha}^{n+1} \Gamma_{2}\right. \\
\left.+|u|_{0}^{0}\right\} .
\end{gathered}
$$

Furthermore, if $\widetilde{P} \in \tilde{\mathscr{E}}$, then

$$
|\widetilde{P} u|_{\alpha}^{n+1} \leqq C\left\{|f|_{l-2 m+\alpha}^{n+1}+\Sigma\left|\varphi_{j}\right|_{l-m_{j}+\alpha}^{q \Gamma_{1}-I_{2}}+\Sigma\left|\varphi_{j}\right|_{l-m_{j}+\alpha}^{n+1} \Gamma_{2}+|u|_{0_{0}}^{0}\right\} .
$$

Proof. We first define a third class $\mathscr{E}_{0}$ consisting of all operators $P(x, D)$ with the same properties a those in $\mathscr{E}$, except that the coefficients need not be differentiable in any tangential direction. Nevertheless, they are to be in $\mathscr{C}_{a}^{n+1}$. In other words, when subjected to any $\mathscr{T}_{y}$, the coefficients are to be in $\mathscr{C}_{a}^{n+1}$ and to have Hölder continuous derivatives with respect to $\bar{x}_{n+1}$. The corresponding norms are to be bounded by $H$. Let $\delta$ be a number to be defined later, and define

$$
M=4 \delta^{-\alpha} \operatorname{lub}_{\mathscr{E}_{0}}|P(x, D) u|_{0}^{0, \mathscr{D}_{1}}+\operatorname{lub}_{\mathscr{E}}|P(x, D) u|_{\alpha}^{q, \mathscr{D}_{1}}+\operatorname{lub}_{\tilde{\mathscr{E}}}|\widetilde{P} u|_{\alpha}^{n+1}
$$

Then there is a point $y \in \mathscr{D}$ and an operator $Q(x, D) \in \mathscr{E}$ (or $\mathscr{E}_{0}$ ) or an operator $\widetilde{Q}(x, D) \in \tilde{\mathscr{E}}$ such that one of the following four quantities is $>\frac{1}{8} M$ :

$$
\begin{aligned}
& U_{1}=4 \delta^{-\alpha}|Q(y, D) u(y)|, \\
& U_{2}=\frac{|Q(z, D) u(z)-Q(y, D) u(y)|}{|z-y|^{\alpha}}
\end{aligned}
$$

(for some $z \in \mathscr{D}_{1}$ with $\hat{\bar{z}}=\hat{\bar{y}}$ for every transformation $\mathscr{T}_{x}$ ),

$$
\begin{aligned}
& U_{3}=|\widetilde{Q}(y, D) u(y)|, \\
& U_{4}=\frac{|\widetilde{Q}(z, D) u(z)-\widetilde{Q}(y, D) u(y)|}{|z-y|^{\alpha}} \quad \text { (for some } z \in \mathscr{D} \text { ). }
\end{aligned}
$$

If $y \notin \mathscr{D}_{1}, U_{1}$ and $U_{2}$ are missing from the list, and if $q=0, U_{2}$ and $U_{4}$ are missing.

First we assume $q>0, y \in \mathscr{D}_{1}, Q \in \mathscr{E}$, and $U_{2}>\frac{1}{8} M$. It may be assumed that $z \in N_{y}$ and that $|z-y| \leqq \delta$ (see the proof to Theorem 5.1). Subject the coordinates to the transformation $\mathscr{T}_{y}$. Then $|\bar{z}-\bar{y}| \leqq$ $\delta^{\prime}=(1 / \kappa) \delta$, where $\kappa$ is the minimum expansion coefficient for the transformations $\mathscr{T}_{y}$. We assume $\delta$ to be small enough so that $\delta^{\prime} \leqq$ $\frac{1}{8}$. Define 
(5.35a)

$$
w(\bar{x})=\zeta\left(\frac{\bar{x}-\bar{y}}{\delta^{\prime}}\right) u(\bar{x}),
$$

if both $\bar{y}$ and $\bar{z}$ are further than $2 \delta^{\prime}$ from $\dot{\mathscr{H}}_{0}$; and

$$
w(\bar{x})=\zeta\left(\frac{\bar{x}-\bar{y}_{B}}{3 \delta^{\prime}}\right) u(\bar{x})
$$

if not (here $\bar{y}_{B}$ is the projection of $\bar{y}$ onto $\dot{\mathscr{C}}_{0}$ ). Since $|\bar{y}| \leqq \frac{1}{3}$ and $|\bar{y}-\bar{z}| \leqq \frac{1}{9}$ it follows that $w$ always has support in $\mathscr{P}$.

We assume alternative (5.35b) as the proof for the other is similar. It is seen from (5.25), (5.27) that after transformation the operator $Q$ may be written as

$$
\bar{Q}(\bar{y}, \bar{D})=Q_{1}(\bar{y}, \bar{D})+Q_{2}(\bar{y}, \bar{D}),
$$

where

$$
Q_{1}(\bar{y}, \bar{D})=\frac{A(\bar{y}, \bar{D})}{\lambda\left(\bar{y}_{B}\right)} L_{y}^{\prime}\left(\bar{y}_{B}, \bar{D}\right)+\sum_{j} \frac{a_{j}(\bar{y}, \bar{D})}{\beta_{j}\left(\bar{y}_{B}\right)} B_{j y}^{\prime}(\bar{y}, \bar{D})+\widetilde{Q}_{3}(\bar{y}, \bar{D}),
$$

where $L_{y}^{\prime}$ and $B_{j y}^{\prime}$ are those parts of $L_{y}$ and $B_{j y}$ consisting of highest order terms only, and $Q_{2}$ vanishes for $\bar{y}_{n+1}=0$. Then

$$
\frac{1}{8} M \leqq T_{1}+T_{2}+T_{3} \text {, }
$$

where

$$
\begin{aligned}
T_{1} & =\frac{\mid Q(\bar{z}, \bar{D})-Q(\bar{y}, \bar{D})) u(\bar{z}) \mid}{|\bar{z}-\bar{y}|^{\alpha}}, \\
T_{2} & =\frac{\left|Q_{1}(\bar{y}, \bar{D}) u(\bar{z})-Q_{1}(\bar{y}, \bar{D}) u(\bar{y})\right|}{|\bar{z}-\bar{y}|^{\alpha}}, \\
T_{3} & =\frac{\left|Q_{2}(\bar{y}, \bar{D}) u(\bar{z})-Q_{2}(\bar{y}, \bar{D}) u(\bar{y})\right|}{|\bar{z}-\bar{y}|^{\alpha}} .
\end{aligned}
$$

"The smoothness of the coefficients of operators in $\mathscr{E}$ tells us

$$
T_{1} \leqq C M \text {. }
$$

Theorem 4.4 (4.18), with the aid of (5.36), may be applied to yield

$$
\begin{aligned}
T_{2} & =\frac{\left|Q_{1}(\bar{y}, \bar{D}) w(\bar{z})-Q_{1}(\bar{y}, \bar{D}) w(\bar{y})\right|}{|\bar{z}-\bar{y}|^{\alpha}} \\
& \leqq C\left\{\left[L_{y}^{\prime}\left(\bar{y}_{B}, \bar{D}\right) w\right]_{l-2 m+\alpha}^{n}+\sum_{j}\left[B_{j y}^{\prime}\left(\bar{y}_{B}, \bar{D}\right) w\right]_{l-m_{j}+\alpha}^{q}\right\} .
\end{aligned}
$$

Let $S_{\delta}$, be the sphere of radius $6 \delta^{\prime}$ about $y_{B}$, so the support of $w$ is in $S_{\delta^{\prime}}$. As in (5.14), we have

$$
\bar{D}^{l-2 m} L_{y}^{\prime}\left(\bar{y}_{B}, \bar{D}\right) w=\zeta \bar{D}^{l-2 m} L_{y}^{\prime}\left(\bar{y}_{B}, \bar{D}\right) u+\sum_{k \geqq 1} \gamma_{k}(\bar{x})\left(\bar{D}^{k} \zeta\right) \bar{D}^{l-k} u,
$$


and from (5.25),

$$
\bar{D}_{\bar{x}}^{l-2 m} L_{k}^{\prime}\left(\bar{y}_{B}, \bar{D}\right) u(\bar{x})=\lambda_{y}\left(\bar{y}_{B}\right)\left(\lambda_{y}(\bar{x})\right)^{-1} \bar{D}_{\bar{x}}^{l-2 m} L_{y}(\bar{x}, \bar{D}) u+\widetilde{Q} *(\bar{x}, \bar{D}) u(\bar{x})
$$

$$
\begin{aligned}
& +\lambda_{y}\left(\bar{y}_{B}\right)\left(\lambda_{y}(\bar{x})\right)^{-1} L_{1 y}(\bar{x}, \bar{D}) \bar{D}^{l-2 m} u(\bar{x}) \\
& + \text { lower order terms }
\end{aligned}
$$

where

$$
\widetilde{Q}^{*}(\bar{x}, \bar{D}) u=\left\{\widetilde{L}_{y}\left(\bar{y}_{B}, \bar{D}\right) \bar{D}^{l-2 m}-\lambda_{y}\left(x_{B}\right)(\lambda(\bar{x}))^{-1} \widetilde{L}_{y}(\bar{x}, \bar{D}) D^{l-2 m}\right\} u .
$$

Since $L_{y}(\bar{x}, \bar{D}) u=f(\bar{x})$ and $\lambda_{y}$ is estimable from above and below in terms of $H$, the first term on the right in (5.40) makes a contribution to $\bar{D}^{l-2 m} L_{y}^{\prime}\left(\bar{y}_{B}, \bar{D}\right) w$ which is estimable in $[\cdot]_{\alpha}^{n}$ norm by $C(\delta)|f|_{l-2 m+\infty}^{n}$. (as in (5.16)). Now $\widetilde{Q}^{*}(\bar{x}, \bar{D})$ is clearly the image under $\mathscr{T}_{y}$ of an operator in $\mathscr{E}$, and furthermore this operator has coefficients bounded in $|\cdot|_{\alpha}^{n+1}$ norm by $C \delta$ for some $C$ depending only on $H$. Therefore, as. in (5.17),

$$
\left.\mid \zeta \widetilde{Q}^{*}(\bar{x}, \bar{D}) u(\bar{x})\right]_{\alpha}^{n+1} \leqq C \delta M+C(\delta)|u|_{0}^{0} .
$$

Thus by continuing the reasoning we obtain

$$
\begin{aligned}
\left.\mid L_{y}^{\prime}\left(\bar{y}_{B}, \bar{D}\right) w\right]_{l-2 m+\alpha}^{n} \leqq C \delta M & +C(\delta)\left\{|f|_{l-2 m+\alpha}^{n}+\Sigma\left|\varphi_{j}\right|_{l-m_{j}}^{0}+|u|_{0}^{0}\right\} \\
& +\left[\zeta \lambda_{y}\left(\bar{y}_{B}\right)\left(\lambda_{y}(\bar{x})\right)^{-1} L_{1 y}(\bar{x}, \bar{D}) \bar{D}^{l-2 m} u\right]_{\alpha}^{u}
\end{aligned}
$$

In the same manner we obtain

$$
\begin{gathered}
{\left[B_{j y}^{\prime}\left(\bar{y}_{B}, \bar{D}\right) w\right]_{l-m_{j}+\alpha}^{q} \leqq C \delta M+C(\delta)\left\{|f|_{l-2 m}^{0}+\Sigma\left|\varphi_{j}(\bar{x})\right|_{l-m_{j}+\alpha}^{q} \dot{\mathscr{H}}_{0}\right.} \\
\left.+|u|_{0}^{0}\right\} .
\end{gathered}
$$

In this latter we use the fact that

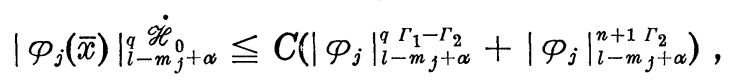

then combine (5.41) and (5.42), estimate $T_{3}$ and the last term in. (5.41) by Lemma 5.2, define $\delta$ to be small enough so that all terms. involving $M$ may be transposed to the left side of the inequality, and (5.31) is proved for this case. A similar proof goes through if the other alternative in (5.35) holds or if $U_{1}, U_{3}$, or $U_{4}$ is $>\frac{1}{8} M$ and $y \in \mathscr{D}_{1}$. Finally, if $y \notin \mathscr{D}_{1}$, the boundary estimates of Agmon, Douglis, and Nirenberg [2, Theorem 7.3] may be applied directly to estimate $U_{3}$ and $U_{4}$ in terms of $|f|_{l-m+\infty}^{n+1} \mathscr{D}_{2}$ and $\Sigma\left|\varphi_{j}\right|_{l-m j+\infty}^{n+1 \Gamma_{2}}$. This completes the proof.

6. The case $q=n$. In this section we shall see that somewhat more concerning equations with variable coefficients may be said when $q=n$ than when $q<n$. In fact, most of the properties of solutions 
of elliptic boundary value problems which are true under complete Hölder-continuity assumptions $(q=n+1)$ of the functions involved are also true (or analogs of them are true) under assumptions corresponding to the case $q=n$. Assuming $q=n$ we shall be able (1) to demonstrate improved versions of Theorems 5.1 and 5.3, and (2) to prove an existence theorem concerning problem (5.1).

First we consider the problem when $\mathscr{D}$ is the half-space $x_{n+1}>0$. The assumption $q=n$ means that all functions concerned are Hölder continuous in all directions except possibly that of the $x_{n+1}$-axis.

THEOREM 6.1. Let $L$ and $B_{j}$ satisfy the ellipticity, complementing, and smoothness conditions stated as hypotheses to Theorem 5.1. If $u(x) \in \mathscr{C}_{l+\alpha}^{n}, f(x) \in \mathscr{C}_{l-2 m+\alpha}^{n}, \varphi_{j}(x) \in \mathscr{C}_{l-m_{j}+\alpha}^{n}$, satisfy (5.1) for $x_{n+1}>$ 0 , then

$$
|u|_{l+\alpha}^{n}+|\tilde{u}|_{l+\alpha}^{n+1}+\leqq C\left\{|f|_{l-2 m+\alpha}^{n}+\Sigma\left|\varphi_{j}\right|_{l-m,+\alpha}^{n}+|u|_{0}^{0}\right\} .
$$

Proof. We shall first show that without any further hypotheses, $L$ and $B_{j}$ may be put into the form (5.2), (5.3), and that the corresponding set $\mathscr{E}$ includes all derivatives $D^{\imath}$. Then an estimate of the form (6.1), with, however, $|f|_{l-2 m+\infty}^{n+1}$ replacing $|f|_{l-2 m+\infty}^{n}$ on the right, clearly follows immediately from (5.5). With no loss of generality we may assume the coefficient of $\partial^{2 m} / \partial x_{n+1}^{2 m}$ in $L(x, D)$ to be identically 1 . Then setting $L_{0}(D)=\partial^{2 m} / \partial x_{n+1}^{2 m}$ and $L_{1}=0,(5.2)$ is obtained. Since the complementing condition assures us that in each $B_{j}$ there is a derivative $\partial^{m_{j}} / \partial x_{n+1}^{m_{j}}$ with non-vanishing coefficient, we do the same thing here $\left(B_{j_{0}}=\partial^{m_{j}} / \partial x_{n+1}^{m_{j}}\right)$. Also every derivative $D^{l}$ is trivially of the form (5.4) with, in fact, $P_{1}=0$ and $a_{j}=0$, so is contained in $\mathscr{E}$.

Lastly we must show that (6.1) is correct as it stands, rather than with $|f|_{l-2 m+\infty}^{n+1}$ on the right. To do this we refer to the proof of Theorem 5.1, in particular to (5.19). At that stage the proper superscript $n$ appears on the right, but it is changed to $n+1$ when the last two terms are estimated (by means of Lemma 5.2). In the present case, however, these last two terms are absent (we have mentioned that $L_{1}=0$, and $T_{3}$ is absent because $Q_{2}=0$ in (5.10)), so that the superscript $n$ remains, and (6.1) is valid.

We now pass to the analog of Theorem 5.3. In that theorem $q$ families of distinguished curves were assumed to cover $\mathscr{D}_{1}$, a portion of $\mathscr{D}$. It will be more convenient in the present case to speak of a one-parameter family of $n$-dimensional hypersurfaces covering $\mathscr{D}_{1}$, the boundary portion $\Gamma_{1}=\dot{\mathscr{D}}_{1} \cap \dot{\mathscr{D}}$ being one of this family. This amounts to the same thing, and the proof is unchanged; moreover this permits the inclusion of the important case when $\Gamma_{1}=\dot{\mathscr{D}}$ but 
$\dot{\mathscr{D}}$ may not be covered with an $n$-parameter family of curves with the required regularity properties holding everywhere (for example, when $\mathscr{D}$ is a sphere in 3-space). Along with this change in point. of view, assumption (2) preceding Theorem 5.3 should be changed to require that these $n$-dimensional hypersurfaces be mapped onto hyperplanes $\bar{x}_{n+1}=$ const. We shall discard hypothesis (4) altogether. Lastly we define a new domain $\mathscr{D}_{2}$ with the properties that $\mathscr{D}_{1} \cup$ $\mathscr{D}_{2}=\mathscr{D}, \dot{\mathscr{D}}_{2} \cap \dot{\mathscr{D}}=\Gamma_{2}$. If $\Gamma_{1}=\dot{\mathscr{D}}, \Gamma_{2}=0$ and $\mathscr{D}_{2}$ is an interior domain.

Theorem 6.2. Let $\mathscr{D}, \mathscr{D}_{1}, \mathscr{D}_{2}, L$, and $B_{j}$ satisfy the hypotheses of Theorem 5.3, with the above modifications, and excluding (5.25), (5.26). Then if $u(x), f(x), \varphi_{j}$ satisfy (5.1) and have the required. smoothness properties,

$$
|\tilde{u}|_{l+\alpha}^{n+1}+|u|_{l+\infty}^{n \cdot \mathscr{D}_{1}} \leqq C\left\{|f|_{l-2 m+\infty}^{n} \mathscr{\mathscr { D }}_{1}+|f|_{l-2 m+\infty}^{n+1, \mathscr{D}_{2}}+\Sigma\left|\varphi_{j}\right|_{l-m}^{n} \underset{l-m}{\dot{\mathscr{D}}+\alpha}+|u|_{0}^{0}\right\}
$$

Proof. Since $q=n$, any operators $L$ and $B_{j}$ automatically satisfy (5.25) and (5.26); and in fact with $L_{1 y}=0$. Also clearly any derivative $D^{\imath}$ is in $\mathscr{E}$, and any such directional derivative involving a differentiation in a direction tangent to a distinguished hypersurface is in $\tilde{\mathscr{E}}$. Hence (6.2) would immediately follow from Theorem 5.3 if the first two terms on the right were replaced by $|f|_{l-2 m+\infty}^{n+1}$. But as in Theorem 6.1, the fact that $L_{1 y}=0$ and that $P_{1 y}$ is not needed in (5.27) results in our not having to require $D^{\imath-2 m} f$ to be Hölder continuous in the one undistinguished direction, for points in $\mathscr{D}_{1}-\mathscr{D}_{2}$. This completes the proof. This theorem is analogous to Theorem 7.3 of [2].

The domain $\mathscr{D}_{2}$ was introduced not only for greater generality, but also because in general such a domain would be needed for topological reasons; it is not always possible to cover the entire domain $\mathscr{D}$ with a regular family of hypersurfaces, one of which is $\dot{\mathscr{D}}$. It is therefore important that such a covering be resticted to $\mathscr{D}_{1}$. However the theorem may be improved to the extent that $f$ still need not be fully Hölder continuous in $\mathscr{D}_{2}$ If there is a second family of distinguished hypersurfaces covering $\mathscr{D}_{2}$ in a regular manner, and not necessarily fitting in with the first family in $\mathscr{D}_{1} \cap \mathscr{D}_{2}$, then the second term on the right of (6.2) may be replaced by $|f|_{l-2 m+\alpha}^{n} \mathscr{D}_{2}$, which is of course to be understood as defined with reference to the second family. The proof offers no difficulties but we shall not give it.

Our final task will be to prove that a solution to the basic problem (5.1) may be expected to exist under the smoothness hypotheses 
corresponding to $q=n$, provided one exists under the stronger hypotheses corresponding to $q=n+1$. But first we consider questions of uniqueness. It is clear from the remark on page 517 that if $f \in \mathscr{C}_{l-2 m+\alpha}^{n+1}(\mathscr{D})$ (and $\varphi_{j} \in \mathscr{C}_{l-m,+\alpha}^{n}(\dot{\mathscr{D}})$ ) then any solution $u \in \mathscr{C}_{l+\alpha}^{n}\left(\mathscr{D}_{1}\right)$ $\cap \mathscr{C}_{l+\alpha}^{n+1}\left(\mathscr{D}_{2}\right)$ to $(5.1)$ is in $\mathscr{C}_{l+\alpha}^{n+1}(\mathscr{D})$. For any $l$ th order directional derivative $\widetilde{D}^{l} u$ written in terms of local coordinate system, which involves a differentiation in a distinguished direction is completely Hölder continuous; but then the only derivative $D^{l}$ not involving such a direction is also completely Hölder continuous, for it may be expressed by means of the differential equation in terms of $f$ and derivative $\widetilde{D}^{l}$. Hence under the hypotheses of Theorem 6.2, if problem (5.1) has at most one solution in $\mathscr{C}_{l+\alpha}^{n+1}(\mathscr{D})$ for every $f \in \mathscr{C}_{l-2 m+\alpha}^{n+1}(\mathscr{D})$, then it has at most one solution $u \in \mathscr{C}_{l+\alpha}^{n}\left(\mathscr{D}_{1}\right) \cap \mathscr{C}_{l+a}^{n+1}\left(\mathscr{D}_{2}\right)$ for every $f \in \mathscr{C}_{l-2 m+\alpha}^{n} \cap \mathscr{C}_{l-2 m+\alpha}^{n+1}\left(\mathscr{D}_{2}\right)$.

THEOREM 6.3. If uniqueness holds in problem (5.1) with $q=n$, then the term $|u|_{0}^{0}$ may be omitted from (6.2).

Proof. If this were not true, there would be a sequence $u^{\nu}$ of functions in $\mathscr{C}_{l+\alpha}^{n}\left(\mathscr{D}_{1}\right) \cap \mathscr{C}_{l+\alpha}^{n+1}\left(\mathscr{D}_{2}\right)$ with $\left|L u^{\nu}\right|_{l-2 m+\alpha}^{n+1} \mathscr{D}_{2},|L u|_{l-2 m+\alpha}^{n} \mathscr{D}_{1}$, and $\left|B_{j} u^{\nu}\right|_{l-m_{j}+\alpha}^{n} \dot{\mathscr{D}}$ bounded, but $\left|u^{\nu}\right|_{0}^{0}$ and $|\tilde{u}|_{l+\alpha}^{n+1}$ or $|u|_{l+\alpha}^{n} \underset{D_{1}}{\mathscr{D}} \rightarrow \infty$. Define the new sequence $\bar{u}^{\nu}=u^{\nu} /\left|u^{\nu}\right|_{0}^{0}$. Then

$$
\left|L \bar{u}^{\nu}\right|_{l-2 m+a}^{n} \mathscr{D}_{1}+\left|L \bar{u}^{\nu}\right|_{l-2 m+\alpha}^{n+1} \mathscr{D}_{2}+\Sigma\left|B_{j} \bar{u}^{\nu}\right|_{l-m_{j}+a}^{n} \underset{\mathscr{D}}{\dot{D}} \rightarrow 0,
$$

but $\left|\bar{u}^{\nu}\right|_{0}^{0}=1$, and according to (6.2), $\left|\tilde{\bar{u}}^{\nu}\right|_{l+\infty}^{n+1}$ and $\left|\bar{u}^{\nu}\right|_{l+\infty}^{n} \mathscr{D}_{1}$ are bounded. From this last fact we know the derivatives of the form $\widetilde{D}^{l} \bar{u}^{\nu}$ to be equicontinuous, and there is a subsequence $\bar{u}^{k} \rightarrow \bar{u}$ with $\widetilde{D}^{\imath} \bar{u}^{k} \rightarrow \widetilde{D}^{l} \bar{u}$, and $D^{\lambda} \bar{u}^{l} \rightarrow D^{\lambda} \bar{u}, \lambda \leqq l-1$, all these convergence processes being uniform. Write $L$ in terms of local coordinates $\bar{x}_{j}$, where $\tilde{\bar{x}}=$ $\left(\bar{x}_{1}, \cdots, \bar{x}_{n}\right)$. Then if $a(\bar{x})$ is the coefficient of $\partial^{2 m} / \partial \bar{x}_{n+1}^{2 m}$ in this expression, we have $\partial^{l} \bar{u}^{k} / \partial \bar{x}_{n+1}^{l}=\Sigma$ (coeffs.) $\widetilde{D}^{l} \bar{u}^{k}+$ lower order terms + $(a(\bar{x}))^{-1}\left(\partial^{l-2 m} / \partial \bar{x}_{n+1}^{l-2 m}\right) L \bar{u}^{k}$. Since the last term approaches 0 as $k \rightarrow \infty$, $\partial^{l} \bar{u}^{k} / \partial x_{n+1}$ converges uniformly to a function, which will therefore be $\partial^{l} \bar{u} / \partial \bar{x}_{n+1}^{l}$, and $\bar{u}$ will satisfy

$$
\begin{aligned}
L \bar{u} & =0 \text { in } \mathscr{D}, \\
B_{j} \bar{u} & =0 \text { on } \dot{\mathscr{D}} .
\end{aligned}
$$

But $\bar{u} \not \equiv 0$, which contradicts the uniqueness assumption, and the theorem is proved.

THEOREM 6.4. Let $\mathscr{D}, \mathscr{D}_{1}, \mathscr{D}_{2}, L$, and $B_{j}$ satisfy the hypotheses of Theorem 6.2. Suppose the problem (5.1) has a unique solution 
$u \in \mathscr{C}_{l+a}^{n+1}(\mathscr{D})$ for every $f \in \mathscr{C}_{l-2 m+a}^{n+1}(\mathscr{D})$ and $\varphi_{j} \in \mathscr{C}_{l-m_{j}+a}^{n}(\dot{\mathscr{D}})$. Then it has a unique solution $u \in \mathscr{C}_{l+a}^{n}\left(\mathscr{D}_{1}\right) \cap \mathscr{C}_{l+a}^{n+1}\left(\mathscr{D}_{2}\right)$ for every $f \in$ $\mathscr{C}_{l-2 m+\alpha}^{n}\left(\mathscr{D}_{1}\right) \cap \mathscr{C}_{l-2 m \alpha}^{n+1}\left(\mathscr{D}_{2}\right)$ and $\varphi_{j} \in \mathscr{C}_{l-m,+\alpha}^{n}(\dot{\mathscr{D}})$.

Proof. Given any $f \in \mathscr{C}_{l-2 m+\alpha}^{n}\left(\mathscr{D}_{1}\right) \cap \mathscr{C}_{l-2 m+\alpha}^{n+1}\left(\mathscr{D}_{2}\right)$, let the family $f_{\varepsilon}(x)$ be made up of functions in $\mathscr{C}_{l-2 m+\infty}^{n+1}(\mathscr{D})$ such that as $\varepsilon \rightarrow 0$, $D^{\lambda} f_{\varepsilon} \rightarrow D^{\lambda} f, \lambda \leqq l-2 m$, for every point in $\mathscr{D}$ at which the latter derivatives are continuous; and $\left|f_{\varepsilon}\right|_{l-2 m+\alpha}^{n} \mathscr{D}_{1} \rightarrow|f|_{l-2 m+\alpha}^{n} \mathscr{D}_{1}$. For example, we could set $f_{\varepsilon}(x)=j_{\varepsilon} * f$ with $j_{\varepsilon}$ as defined in the proof to Theorem 4.6. By assumption and Theorems 6.2, 6.3, for each $\varepsilon$ there is a unique $u_{\varepsilon}(x) \in \mathscr{C}_{l+\alpha}^{n+1}(\mathscr{D})$ such that

$$
\begin{aligned}
L u_{\varepsilon} & =f_{\varepsilon} \text { in } \mathscr{D}, \\
B_{j} u_{\varepsilon} & =\varphi_{j} \text { on } \dot{\mathscr{D}},
\end{aligned}
$$

and

$$
\left|\widetilde{u}_{\varepsilon}\right|_{l+\alpha}^{n+1, \mathscr{D}}+\left|u_{\varepsilon}\right|_{l+a}^{n \mathscr{D}} \mathscr{D}_{1} \leqq C\left\{\left|f_{\varepsilon}\right|_{l-2 m+\alpha}^{n} \mathscr{D}_{1}+\left|f_{\varepsilon}\right|_{l-2 m+\alpha}^{n+1} \mathscr{D}_{2}+\Sigma\left|\varphi_{j}\right|_{l-m, j+\alpha}^{n}\right\} .
$$

Hence the norms $\left|\widetilde{u}_{\varepsilon}\right|_{l+\infty}^{n+1, \mathscr{D}}$ and $\left|u_{\varepsilon}\right|_{l+a}^{n} \mathscr{D}_{1}$ form bounded sets. We shall show that the set of functions $u_{\varepsilon}$ is compact in $\mathscr{C}_{l+\infty}^{n}\left(\mathscr{D}_{1}\right)$. The boundedness of the norms $\left|\widetilde{u}_{\varepsilon}\right|_{l+\alpha}^{n+1}$, shows the set of derivatives $\widetilde{D}^{l} u_{\varepsilon}$ to be equicontinuous. Denoting by $\mathscr{D}_{\delta}$ the portion of $\mathscr{D}_{1}$ that remains after a $\delta$-neighborhood of every point of discontinuity of $D^{l-2 m} f$ has been deleted, it is clear that the $D^{l-2 m} f_{\varepsilon}$ will be equicontinuous in $\mathscr{D}_{\delta}$. Solving the differential equation for $\partial^{l} u_{\varepsilon} /\left(\partial \bar{x}_{n+1}\right)^{l}$, the only $l$ th order derivative not of the form $\widetilde{D}^{l} u_{\varepsilon}$, we see that it, hence all $D^{l} u_{\varepsilon}$, are equicontinuous in $\mathscr{D}_{\delta}$, and a subsequence of the $u_{\varepsilon}$ converges to a function $u_{\delta}$ which satisfies the differential equation $L u_{\delta}=f$ in $\mathscr{D}_{\delta}$, and the boundary conditions $B_{j} u_{\delta}=\varphi_{j}$ on $\dot{\mathscr{D}}$. Now taking a sequence of positive numbers $\delta_{\nu} \rightarrow 0$ and a diagonal subsequence of the $u_{\varepsilon}$, we find that the latter converges to a function $u \in \mathscr{C}_{l+\alpha}^{n}\left(\mathscr{D}_{1}\right) \cap \mathscr{C}_{l+a}^{n+1}\left(\mathscr{D}_{2}\right)$ which satisfies (5.1).

APPENDIX A. Proof of Lemma 4.3. What we shall show specifically is that if the contour $\gamma$ and function $F(\xi, \tau)$ are as in Lemma $4.1, k>q$, and $F$ is differentiable with respect to $\xi$ for $\tau \in \gamma,|\xi|=$ 1 , then

$$
\begin{aligned}
& \int_{\tilde{x}-\text { space }} d \tilde{x} \int_{|\xi|=1} d \omega_{\xi} \int_{\gamma} F(\tilde{\xi}, \hat{\xi} ; \tau)(x \cdot+\xi+t \tau)^{-k} d \tau \\
& \quad=\frac{(2 \pi i)_{q}(k-q-1) !}{(k-1) !} \int_{|\hat{\xi}|=1} d \omega_{\hat{\xi}} \int_{\gamma} F(0, \hat{\xi} ; \tau)(\hat{x} \cdot \hat{\xi}+t \tau)^{-k+q} d \tau .
\end{aligned}
$$

With this established, (4.9) will follow as a special case, in view of 
(4.6) (see the definition of $b_{j, s}$ in [2]). The lemma tells us nothing new in case $q=0$. First we prove it for the case $q=1, \widetilde{x}=x_{1}$; an obvious iteration process will then yield the result for the general case. First we recognize that

$$
\begin{aligned}
& \int d \omega_{\xi} \int_{\gamma} F(\xi, \tau)(x \cdot \xi+t \tau)^{-k} d \tau \\
& \quad=\frac{1}{1-k} \frac{\partial}{\partial x_{1}} \int_{\substack{|\xi|=1 \\
P V}} d \omega_{\xi} \int_{\gamma} \frac{1}{\xi_{1}} F(\xi, \tau)(x \cdot \xi+t \tau)^{-k+1} d \tau,
\end{aligned}
$$

where " $P V$ " indicates that the integral is taken in the principal value sense. This is easily checked by forming the derivative as the limit of difference quotients. Letting $I$ denote the left side of (A.1), it follows that

$$
I=\frac{1}{1-k}\left\{\int_{\substack{i \xi^{\prime}=1 \\ P V}} d \omega_{\xi} \int_{\gamma} \frac{1}{\xi_{1}} F\left(\xi_{1}, \hat{\xi} ; \tau\right)(x \cdot \xi+t \tau)^{1-k} d \tau\right\}_{x_{1}=-\infty}^{x_{1}=+\infty} .
$$

Now let $\zeta_{\delta}\left(\xi_{1}\right)$ be an infinitely differentiable function depending on a small parameter $\delta>0$, such that

$$
\begin{aligned}
& \zeta_{\delta}\left(\xi_{1}\right)=0 \text { for }\left|\xi_{1}\right|>1-\delta \\
& \zeta_{\delta}\left(\xi_{1}\right)=1 \text { for }\left|\xi_{1}\right|<1-2 \delta .
\end{aligned}
$$

The principal value integral (A.2) may be converted into an ordinary integral by subtracting from the coefficient of $\xi_{1}^{-1}$ in the integrand any even smooth function of $\xi_{1}$ which takes on the same value as the original coefficient when $\xi_{1}=0$. For this function we choose

$$
F\left(0, \hat{\xi}\left(1-\xi_{1}^{2}\right)^{-1 / 2} ; \tau\right) \zeta_{\delta}\left(\xi_{1}\right)\left(1-\xi_{1}^{2}\right)^{(-n+3) / 2}\left(\hat{x} \cdot \hat{\xi}\left(1-\xi_{1}^{2}\right)^{-1 / 2}+t \tau\right)^{1-k} .
$$

Carrying out the subtraction in three parts, we obtain $I=I_{1}+I_{2}+$ $I_{3}$, where

$$
\begin{gathered}
I_{1}=\frac{1}{1-k}\left\{\int_{|\xi|=1} d \omega_{\xi} \int_{\gamma} \frac{F\left(\xi_{1}, \hat{\xi} ; \tau\right)-F\left(0, \hat{\xi}\left(1-\xi_{1}^{2}\right)^{-1 / 2} ; \tau\right) \zeta_{\delta}\left(\xi_{1}\right)\left(1-\xi_{1}^{2}\right)^{(-n+3) / 2}}{\xi_{1}} \cdot\right. \\
I_{2}=\frac{1}{1-k}\left\{\int_{|\xi|=1} d \omega_{\xi} \int_{\gamma} F\left(0, \hat{\xi}\left(1-\xi_{1}^{2}\right)^{-2 / 2} ; \tau\right) \zeta_{\delta}\left(\xi_{1}\right)\left(1-\xi_{1}^{2}\right)^{(-n+3) / 2} \cdot\right. \\
\left.\cdot \frac{(x \cdot \xi+t \tau)^{1-k}-\left(x_{1} \xi_{1}+\hat{x} \cdot \hat{\xi}\left(1-\xi_{1}^{2}\right)^{-1 / 2}+\tau t\right)^{1-k}}{\xi_{1}} d \tau\right\}_{-\infty}^{+\infty}, \\
I_{3}=\frac{1}{1-k}\left\{\int_{|\xi|=1} d \omega_{\xi} \int_{\gamma}^{x_{1}=+\infty} F\left(0, \hat{\xi}\left(1-\xi_{1}^{2}\right)^{-1 / 2} ; \tau\right) \zeta_{\delta}\left(\xi_{1}\right)\left(1-\xi_{1}^{2}\right)^{(-n+3) / 2} \cdot\right. \\
\left.\quad \frac{\left(x_{1} \xi_{1}+\hat{x} \cdot \hat{\xi}\left(1-\xi_{1}^{2}\right)^{-1 / 2}+\tau t\right)^{1-k}-\left(\hat{x} \cdot \hat{\xi}\left(1-\xi_{1}^{2}\right)^{-1 / 2}+t \tau\right)^{1-k}}{\xi_{1}} d \tau\right\}_{-\infty}^{+\infty}
\end{gathered}
$$


The coefficient of $(\cdots)^{1+k}$ in the integrand of $I_{1}$ is a continuous and bounded function of $\xi$ on $|\xi|=1$; hence by Lemma 4.1 the integral is bounded by $C\left(|x|^{2}+t^{2}\right)^{1-k}$ (C possibly depending on $\delta$ ), which approaches 0 as $x_{1} \rightarrow \pm \infty$. Hence $I_{1}=0$.

Next we use the mean value theorem to write the fraction in the integrand of $I_{2}$ as

$$
\frac{\left(1-\xi_{1}^{2}\right)^{-1 / 2}-1}{\xi_{1}} \hat{x} \cdot \hat{\xi}(k-1)\left(x_{1} \xi_{1}+\hat{x} \cdot \hat{\xi}\left(1-\xi^{2}\right)^{-1 / 2}+t \tau\right)^{-k},
$$

and observe that

$$
\frac{\left(1-\xi_{1}^{2}\right)^{-1 / 2}-1}{\xi_{1}}<\xi_{1} \quad \text { for } \xi_{1}<1 / 2 \text {. }
$$

It is then easy to see that for $x_{1}$ large enough and $\xi_{1} \leqq\left|x_{1}\right|^{-1 / 2}$, the integrand of $I_{2}$ is bounded by $C(\widehat{x}, t)\left|x_{1}\right|^{-1 / 2}$ independently of $\hat{\xi}$ and $\tau$. Also for $\xi_{1} \geqq\left|x_{1}\right|^{-1 / 2}$ and $x_{1}$ large enough the integrand is bounded by $C(\delta, \hat{x}, t)\left|x_{1} \xi_{1}\right|^{-k} \leqq C(\delta, \hat{x}, t)\left|x_{1}\right|^{-1}$ (since $k \geqq 2$ ). Both of these bounds approach zero as $x_{1} \rightarrow \pm \infty$, so we conclude that $I_{2}=0$.

To analyze $I_{3}$ we use the fact that

$$
\int_{|\xi|=1} G\left(\xi_{1}, \hat{\xi}\right) d \omega_{\xi}=\int_{-1}^{1} d \xi_{1} \int_{|\hat{\xi}|=1} G\left(\xi_{1}, \hat{\xi}\left(1-\xi_{1}^{2}\right)^{1 / 2}\right)\left(1-\xi_{1}^{2}\right)^{(n-3) / 2} d \omega_{\hat{\xi}},
$$

and obtain, after rearranging terms,

$$
\begin{aligned}
I=I_{3}= & \frac{1}{1-k} \int_{|\hat{\xi}|=1} d \omega_{\hat{\xi}} \int_{\gamma} F(0, \xi ; \tau) . \\
& \cdot \sum_{r=1}^{k-1}(\hat{x} \cdot \hat{\xi}+t \tau)^{-r}\left\{\int_{-1}^{1} x_{1}(x \cdot \xi+t \tau)^{r-k} \zeta_{\delta}\left(\xi_{1}\right) d \xi_{1}\right\}_{x_{1}=-\infty}^{x_{1}=+\infty} d \tau .
\end{aligned}
$$

Now changing to a new integration variable $u=x_{1} \xi_{1}$,

$$
\begin{aligned}
\int_{-1}^{1} x_{1}(x \cdot \xi+t \tau)^{r-k} \zeta_{\delta}\left(\xi_{1}\right) d \xi_{1} & =\int_{-x_{1}}^{x_{1}}(u+\hat{x} \cdot \hat{\xi}+t \tau)^{r-k} d u \\
& -x_{1} \int_{\left|\xi_{1}\right|>1-2 \delta}\left[1-\zeta_{\delta}\left(\xi_{1}\right)\right]\left(x_{1} \xi_{1}+\hat{x} \cdot \hat{\xi}+t \tau\right)^{r-k} d \xi_{1} .
\end{aligned}
$$

First we estimate the last integral. For $x_{1}$ large enough, $\left|\xi_{1}\right|>1-$ $2 \delta,\left|x_{1} \xi_{1}+\hat{x} \cdot \hat{\xi}+t \tau\right|>x_{1} / 2$, so that the integral is less in absolute value than $2 \delta x_{1}\left(x_{1} / 2\right)^{r-k}=C \delta x_{1}^{r+1-k}$. For $r<k-1$ this vanishes as $x_{1} \rightarrow \pm \infty$, and for $r=k-1$ it remains bounded by $C \delta$. As for the first integral,

$\left.\lim _{x_{1} \rightarrow \pm \infty} \int_{-x_{1}}^{x_{1}}(u+\hat{x} \cdot \hat{\xi}+t \tau)^{r-k} d u=\lim \frac{1}{r+1-k}(u+\hat{x} \cdot \hat{\xi}+t \tau)^{r+1-k}\right]_{-x_{1}}^{x_{1}}=0$

for $r<k-1$. For $r=k-1$ the same integral 


$$
=\lim _{x_{1} \rightarrow \pm \infty} \log \frac{x_{1}+\hat{x} \cdot \hat{\xi}+t \tau}{-x_{1}+\hat{x} \cdot \hat{\xi}+t \tau}=\mp \pi i .
$$

Hence all terms of the summation in (A.3) vanish except the one with $r=k-1$, and

$$
I=\frac{2 \pi_{i}}{k-1} \int_{|\hat{\xi}|=1} d \omega_{\hat{\xi}} \int_{\gamma} F(0, \hat{\xi} ; \tau)(\hat{x}, \hat{\xi}+t \tau)^{1-k} d \tau+0(\delta) .
$$

But since $\delta$ may be arbitrarily small, the term here of order $\delta$ is really zero, and we have proven (A.1) for the case $q=1$. But if $q>1$ the above procedure may be iterated by integrating successively with respect to $x_{1}, \cdots, x_{q}$. Thus Lemma 4.3 is proved.

APPENDIX B. Proof of Theorem 4.3. We shall prove that a necessary condition for (4.16) to hold is that in every representation (4.14), the $a_{j}(\xi)$ satisfy

$$
a_{j}(0,-\hat{\xi})=(-1)^{l-m_{j}} a_{j}(0, \hat{\xi}) .
$$

That this implies the condition stated may be demonstrated by setting $a_{0 j}(\hat{\xi})=a_{j}(0, \hat{\xi})$ and showing that there is a polynomial $A_{0}(\hat{\xi}, \tau)$ such that

$$
a(0, \hat{\xi}, \tau) M^{+}(0, \hat{\xi}, \tau)=A_{0}(\hat{\xi}, \tau) L(0, \hat{\xi}, \tau)
$$

Since

$$
P(-\xi,-\tau)=(-1)^{l} P(\xi, \tau), M^{+}(-\xi,-\tau)=(-1)^{m} M^{-}(\xi, \tau)
$$

where $M^{+}(\xi, \tau) M^{-}(\xi, \tau)=L(\xi, \tau)$, and $B_{j}(-\xi,-\tau)=(-1)^{m_{j}} B_{j}(\xi, \tau)$, we have from (4.14) and (B.1)

$$
\begin{aligned}
& a(0, \hat{\xi}, \tau) M^{+}(0, \hat{\xi}, \tau)+\sum_{j} a_{j}(0, \hat{\xi}) B_{j}(0, \hat{\xi}, \tau) \\
& \quad=P(0, \hat{\xi}, \tau)=(-1)^{\imath} P(0,-\hat{\xi},-\tau) \\
& \quad=(-1)^{l+m} a(0,-\hat{\xi},-\tau) M^{-}(0, \hat{\xi}, \tau)+\Sigma \alpha_{j}(0, \hat{\xi}) B_{j}(0, \hat{\xi}, \tau) .
\end{aligned}
$$

Hence

$$
(-1)^{l+m} a(0,-\hat{\xi},-\tau) M^{-}(0, \hat{\xi}, \tau)=a(0, \hat{\xi}, \tau) M^{+}(0, \hat{\xi}, \tau) .
$$

But for $\hat{\xi} \neq 0, M^{+}(0, \hat{\xi}, \tau)$ and $M^{-}(0, \hat{\xi}, \tau)$, as polynomials in $\tau$, have no factors in common; hence $M^{-}(0, \hat{\xi}, \tau)$ must divide $a(0, \hat{\xi}, \tau)$ :

$$
a(0, \hat{\xi}, \tau) M^{+}(0, \hat{\xi}, \tau)=A_{0}(\hat{\xi}, \tau) M-M^{+}=A_{0}(\tilde{\xi}, \tau) L(0, \hat{\xi}, \tau) .
$$

Now we proceed to show that (B.1) is necessary. Assume, on the contrary, that there is a value $\hat{\xi}=\hat{\xi}_{0}$ such that $a_{j}\left(0,-\hat{\xi}_{0}\right) \neq$ $(-1)^{l-m_{j}} a\left(0, \hat{\xi}_{0}\right)$. We shall construct a family of functions $\varphi_{j}^{\varsigma}(x)$ such 
that $\left[\varphi_{j}^{\varepsilon}\right]_{l-m_{j}+\alpha}^{q}$ are bounded as $\varepsilon \rightarrow 0$, whereas $\left[P w^{\varepsilon}\right]_{\alpha}^{q}$ are not (here $w^{\varepsilon}$ is defined in terms of $\varphi_{j}^{\varepsilon}$ by (4.3)). We may rotate the coordinate system so that $\hat{\xi}_{0}$ is directed along the $x_{n}$-axis; also since the $a_{j}$ are homogeneous of degree $l-m_{j}$, it may be assumed that $\left|\hat{\xi}_{0}\right|=1: \hat{\xi}_{0}=$ $(0, \cdots, 0,1)$.

Define

$$
\begin{aligned}
\eta^{\varepsilon}(s) & =0 \quad \text { for } s \leqq-\varepsilon, \\
& =s^{l-m_{j}} \text { for } s \geqq \varepsilon,
\end{aligned}
$$

and smooth it off in the range $-\varepsilon \leqq s \leqq \varepsilon$ so that it is an infinitely differentiable function whose derivatives of orders $\leqq l-m_{j}$ are monotonically increasing in $-\varepsilon \leqq s \leqq \varepsilon$. Our sequence $\varphi_{j}^{\varepsilon}$ will be

$$
\varphi_{j}^{\varepsilon}(x)=\eta^{\varepsilon}\left(x_{n}\right) \zeta(|x|),
$$

where $\zeta(s)$ is a $\mathscr{C}_{\infty}$ function with $\zeta=0$ for $|s|>1$ and $\zeta=1$ for $|s|<1 / 2$. It is easily checked that $\left(\partial^{l-m_{j}} / \partial x_{n}^{l-m_{j}}\right) \varphi_{j}^{\varepsilon}(x)$ is bounded independently of $\varepsilon$; hence so is $\left[\varphi_{j}^{\varepsilon}\right]_{l-m_{j}+\alpha}^{\varepsilon}$. We shall show that $P(D) w^{\text {\& }}$ may be made arbitrarily large by choosing $\varepsilon$ and $t$ small. We assume $l-m_{j}$ to be even; the proof for odd case is similar. At this point we apply representation (2.7) to (4.3) after integrating by parts as before; and for this purpose we redefine $\tilde{x}=\left(x_{1} \cdots, x_{n-1}\right), \widehat{x}=x_{n}$. Hence

$$
\begin{aligned}
P(D) w^{\varepsilon}= & P \Delta^{(1 / 2)\left(n+s-l+m_{j}\right)} K_{j, s} *\left[\Delta^{\left(l-m_{j}\right) / 2} \varphi_{j}^{\varepsilon}(y)-\Delta^{\left(l-m_{j}\right) / 2} \varphi_{J}^{\varepsilon}\left(x_{1}, \cdots, x_{n-1}, y_{n}\right)\right] \\
& +\int_{-\infty}^{\infty} \Delta^{(1 / 2)\left(l-m_{j}\right)} \varphi_{j}^{\varepsilon}\left(x_{1} \cdots x_{n-1}, y_{n}\right) K^{*}\left(x_{n}-y_{n} ; t\right) d y_{n}
\end{aligned}
$$

where

$$
K^{*}\left(x_{n} ; t\right)=\iint_{-\infty}^{\infty} P 4^{(1 / 2)\left(n+s-l+m_{j}\right)} K_{j, s}(x, t) d x_{1} \cdots d x_{n-1} .
$$

Since the behavior of $\varphi_{j}^{\varepsilon}$ with respect to the variables $x_{1}, \cdots, x_{n-1}$ is essentially independent of $\varepsilon$, the bracketed expression in the first term on the right is certainly bounded by const. $|x-y|^{\alpha}$, where the constant is independent of $\varepsilon$. Hence by the methods of Theorem 2.1, this first term and its Hölder difference quotients are bounded by a constant independent of $\varepsilon$. Also Lemma 4.3 with (4.6) and (4.14) tell us

$$
\begin{aligned}
K^{*}\left(x_{n} ; t\right)= & \text { const. } \sum_{\varepsilon_{n}= \pm 1} \int_{\gamma} \frac{P\left(0, \xi_{n}, \tau\right) N_{j}\left(0, \xi_{n}, \tau\right)}{M^{+}\left(0, \xi_{n}, \tau\right)\left[x_{n} \xi_{n}+t \tau\right]} d \tau \\
= & \text { const. } \sum_{\xi_{n}= \pm 1} \int_{\gamma} \frac{P\left(0, \xi_{n}, \tau\right) N_{j}\left(0, \xi_{n}, \tau\right)}{M^{+}\left(0, \xi_{n}, \tau\right)}\left[\left(x_{n} \xi_{n}+t \tau\right)^{-1}\right. \\
& \left.-\left(x_{n} \xi_{n}+t \tau_{0}\right)^{-1}\right] d \tau+
\end{aligned}
$$




$$
\begin{aligned}
& + \text { const. } \sum_{\xi_{n}= \pm 1}\left(x_{n} \xi_{n}+t \tau_{0}\right)^{-1} \int a\left(0, \xi_{n}, \tau\right) N_{j}\left(0, \xi_{n}, \tau\right) \\
& +\frac{\Sigma a_{i}\left(0, \xi_{n}\right) B_{i}\left(0, \xi_{n}, \tau\right) N_{j}\left(0, \xi_{n}, \tau\right)}{M^{+}\left(0, \xi_{n}, \tau\right)} d \tau,
\end{aligned}
$$

where $\tau_{0}$ is a point on $\gamma$ and on the imaginary axis: $\tau_{0}=i\left|\tau_{0}\right|$. The first term on the right may be written as

$$
\text { const. } \sum_{\xi_{n}= \pm 1} t \int_{\gamma} d \tau \int_{\tau_{0}}^{\tau} \frac{P N_{j}}{M^{+}}\left(x_{n} \xi_{n}+t \sigma\right)^{-2} d \sigma,
$$

hence estimated (Lemma 4.1) by const. $t\left(x_{n}^{2}+t^{2}\right)^{-1}$. By virtue of the properties of $N_{j}$, the final term may be expressed as

$$
\begin{aligned}
\sum_{\xi_{n}= \pm 1}\left(x_{n} \xi_{n}+t \tau_{0}\right)^{-1} a_{j}\left(0, \xi_{n}\right)= & a_{j}(0,1)\left\{\left(x_{n}+t \tau_{0}\right)^{-1}+\left(-x_{n}+t \tau_{0}\right)^{-1}\right. \\
& -\left(-x_{n}+t \tau_{0}\right)^{-1}\left(a_{j}(0,1)-a_{j}(0,-1)\right) \\
= & -\frac{2 a_{j}(0,1) \tau_{0} t}{x_{n}^{2}+\left|\tau_{0}\right|^{2} t^{2}}+\frac{\Delta a_{j}}{x_{n}-\tau_{0} t}
\end{aligned}
$$

where $\Delta a_{j}=a_{j}(0,1)-a_{j}(0,-1)$. Thus

$$
K^{*}\left(x_{n} ; t\right)=K_{1}^{*}\left(x_{n} ; t\right)+\text { const. } \frac{\Delta a_{j}}{x_{n}-\tau_{0} t}
$$

where $\left|K_{1}^{*}\right|<$ const. $t\left(x_{n}^{2}+t^{2}\right)^{-1}$. Therefore, using the fact that $\int_{-\infty}^{\infty}\left[t /\left(\left(x_{n}-y_{n}\right)^{2}+t^{2}\right)\right] d y_{n}$ is bounded independently of $t$ and $x_{n}$, and $\Delta^{(1 / 2)\left(l-m_{j}\right)} \varphi_{j}^{\varepsilon}(x)$ is bounded independently of $\varepsilon$, we have (setting $\tilde{x}=0$ )

$$
\begin{aligned}
& P w^{\mathfrak{\varepsilon}}\left(0, x_{n}\right) \\
& =W^{\varepsilon}+\text { const. }\left(\Delta a_{j}\right) \int_{-\infty}^{\infty} \zeta\left(\left|y_{n}\right|\right) \frac{d^{l-m_{j} \eta}\left(y_{n}\right)}{d y_{n}^{l-m_{j}}}\left[\left(x_{n}-y_{n}\right)-t \tau_{0}\right]^{-1} d y_{n},
\end{aligned}
$$

where $\left|W^{\varepsilon}\right|<$ const. (independent of $\varepsilon$ ). Now this last integral may be written as $I_{1}+I_{2}$, the two parts corresponding to the ranges $-\varepsilon<$ $y_{n}<1 / 2$ and $1 / 2<y_{n}<1$ (the integrand vanishes for $y_{n}<-\varepsilon$ and $\left.y_{n}>1\right)$. At this point we set $x_{n}=0$. Then since $\left(d^{l-m_{j}} / d y^{l-m_{j}}\right) \eta^{\varepsilon}\left(y_{n}\right)$ $=\left(l-m_{j}\right)$ ! for $y_{n}>\varepsilon$ and $\left|y_{n}-t \tau_{0}\right|^{-1}<4$ for $y_{n}>1 / 2, I_{2}$ is easily estimated as

$$
\left|I_{2}(0, t)\right|<\text { const. }\left(l-m_{j}\right) !
$$

For $I_{1}$ we obtain

$$
\text { const. } \begin{aligned}
I_{1}(0, t) & =\left(\Delta a_{j}\right) \int_{-\varepsilon}^{1 / 2} D^{l-m_{j}} \eta^{\varepsilon}\left[-y_{n}-t \tau_{0}\right]^{-1} d y_{n} \\
& \left.=-\left(\Delta a_{j}\right)\left(D^{l-m_{j}} \eta\left(y_{n}\right)\right) \log \left(-y_{n}-t \tau_{0}\right)\right]_{-\varepsilon}^{1 / 2}+
\end{aligned}
$$




$$
\begin{aligned}
& +\left(\Delta a_{j}\right) \int_{-\varepsilon}^{1 / 2} D^{l-m_{j}+1} \eta^{\varepsilon} \log \left(-y_{n}-t \tau_{0}\right) d y_{n} \\
= & -\left(\Delta a_{j}\right)\left(l-m_{j}\right) ! \log \left(-1 / 2-t \tau_{0}\right) \\
& +\left(\Delta a_{j}\right) \int_{-\varepsilon}^{\varepsilon} D^{l-m_{j}+1} \eta^{\varepsilon}\left(y_{n}\right) \log \left(-y_{n}-t \tau_{0}\right) d y_{n} .
\end{aligned}
$$

The first term in the last expression is independent of $\varepsilon$ and $t$. By construction, $D^{l-m_{j}+1} \eta^{q}\left(y_{n}\right)$ is a nonnegative function vanishing for $\left|y_{n}\right|>\varepsilon$ such that $\int_{-\varepsilon}^{\varepsilon} D^{l-m_{j}+1} \eta^{\S} d y_{n}=\left(l-m_{j}\right) !$. Since $\tau_{0}$ is imaginary,

$$
-R e \log \left(-y_{n}-t \tau_{0}\right)=\frac{1}{2}\left|\log \left(y_{n}^{2}+\left|t \tau_{0}\right|^{2}\right)\right| \geqq \frac{1}{2}\left|\log \left(\varepsilon^{2}+t^{2}\left|\tau_{0}\right|^{2}\right)\right|
$$

for $t$ and $\varepsilon$ small enough and $\left|y_{n}\right|<\varepsilon$. Therefore the last integral in the expression for $I_{1}(0, t)$ is unbounded as $\varepsilon$ and $t$ approach 0 , and the theorem is proved.

\section{BIBLIOGRAPHY}

1. S. Agmon, Maximum theorems for solutions of higher order elliptic equations, Bull. Amer. Math. Soc., 66, No. 2, (1960), 77-80.

2. S. Agmon, A. Douglis and L. Nirenberg, Estimates near the boundary for solutions of elliptic partial differential equations satisfying general bounday conditions $I$, Comm. Pure Appl. Math., 12 (1959), 623-727.

3. A. Douglis, and L. Nirenberg, Interior estimates for elliptic systems of partial differential equations, Comm. Pure Appl. Math., 8 (1955).

4. F. John, Plane Waves and Spherical Means Applied to Partial Differential Equations, Interscience Publishers, New York, 1955.

5. L. Lichtenstein, Encyklopädie der Mathematischen Wisscnschaften, II. C. 3, pp. 197-217.

6. J. Schauder, Über lineare elliptische Differentialgleichungen zweiter Ordnung, Math. Z., 38 (1934), 257-282.

7. - Numerische Abschätzungen in elliptischen linearen Differentialgleichungen, Studia Math., 5 (1934), 34-42. 


\title{
PACIFIC JOURNAL OF MATHEMATICS
}

\author{
EDITORS
}

\author{
Ralph S. Phillips \\ Stanford University \\ Stanford, California \\ M. G. Arsove \\ University of Washington \\ Seattle 5 , Washington
}

\author{
J. DugunduI \\ University of Southern California \\ Los Angeles 7, California
}

Lowell J. Paige

University of California

Los Angeles 24, California

\section{ASSOCIATE EDITORS}

E. F. BECKENBACH

T. M. CHERRY
D. DERRY
M. OHTSUKA

H. L. ROYDEN

E. SPANIER
E. G. STRAUS

F. WOLF

\section{SUPPORTING INSTITUTIONS}

UNIVERSITY OF BRITISH COLUMBIA CALIFORNIA INSTITUTE OF TECHNOLOGY UNIVERSITY OF CALIFORNIA MONTANA STATE UNIVERSITY UNIVERSITY OF NEVADA NEW MEXICO STATE UNIVERSITY OREGON STATE UNIVERSITY UNIVERSITY OF OREGON OSAKA UNIVERSITY UNIVERSITY OF SOUTHERN CALIFORNIA
STANFORD UNIVERSITY

UNIVERSITY OF TOKYO

UNIVERSITY OF UTAH

WASHINGTON STATE UNIVERSITY

UNIVERSITY OF WASHINGTON

AMERICAN MATHEMATICAL SOCIETY CALIFORNIA RESEARCH CORPORATION SPACE TECHNOLOGY LABORATORIES NAVAL ORDNANCE TEST STATION

Mathematical papers intended for publication in the Pacific Journal of Mathematics should be typewritten (double spaced), and the author should keep a complete copy. Manuscripts may be sent to any one of the four editors. All other communications to the editors should be addressed to the managing editor, L. J. Paige at the University of California, Los Angeles 24, California.

50 reprints per author of each article are furnished free of charge; additional copies may be obtained at cost in multiples of 50 .

The Pacific Journal of Mathematics is published quarterly, in March, June, September, and December. Effective with Volume 13 the price per volume (4 numbers) is $\$ 18.00$; single issues, $\$ 5.00$. Special price for current issues to individual faculty members of supporting institutions and to individual members of the American Mathematical Society: $\$ 8.00$ per volume; single issues \$2.50. Back numbers are available.

Subscriptions, orders for back numbers, and changes of address should be sent to Pacific Journal of Mathematics, 103 Highland Boulevard, Berkeley 8, California.

Printed at Kokusai Bunken Insatsusha (International Academic Printing Co., Ltd.), No. 6, 2-chome, Fujimi-cho, Chiyoda-ku, Tokyo, Japan.

PUBLISHED BY PACIFIC JOURNAL OF MATHEMATICS, A NON-PROFIT CORPORATION

The Supporting Institutions listed above contribute to the cost of publication of this Journal, but they are not owners or publishers and have no responsibility for its content or policies. 


\section{Pacific Journal of Mathematics}

\section{Vol. 13, No. 2 \\ April, 1963}

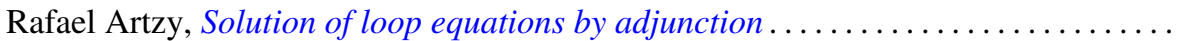

Earl Robert Berkson, A characterization of scalar type operators on reflexive

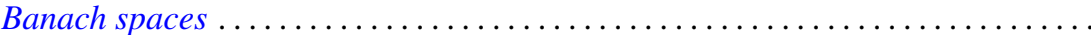

Mario Borelli, Divisorial varieties

365

Raj Chandra Bose, Strongly regular graphs, partial geometries and partially

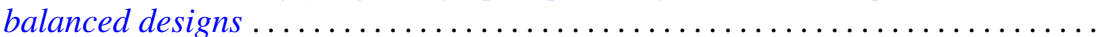

389

R. H. Bruck, Finite nets. II. Uniqueness and imbedding ............... 421

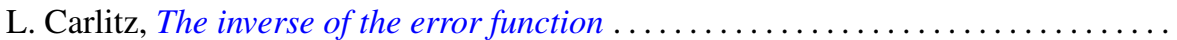

Robert Wayne Carroll, Some degenerate Cauchy problems with operator

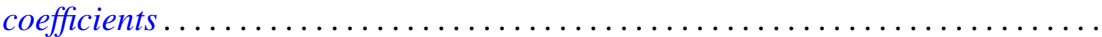

Michael P. Drazin and Emilie Virginia Haynsworth, A theorem on matrices of 0 's

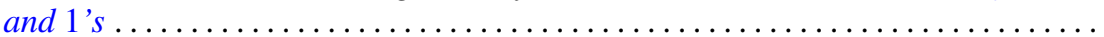

Lawrence Carl Eggan and Eugene A. Maier, On complex approximation .......... James Michael Gardner Fell, Weak containment and Kronecker products of group

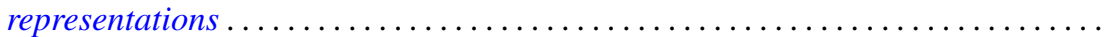

Paul Chase Fife, Schauder estimates under incomplete Hölder continuity

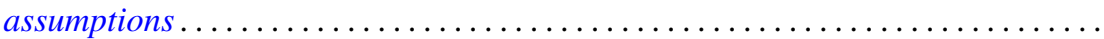

Shaul Foguel, Powers of a contraction in Hilbert space ...................

Neal Eugene Foland, The structure of the orbits and their limit sets in continuous

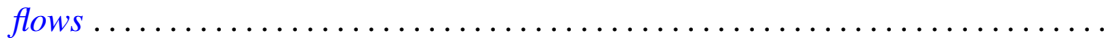

Frank John Forelli, Jr., Analytic measures . . . . . . . . . . . . . . . . . . . . . 563

Robert William Gilmer, Jr., On a classical theorem of Noether in ideal theory ....... P. R. Halmos and Jack E. McLaughlin, Partial isometries .

Albert Emerson Hurd, Maximum modulus algebras and local approximation in

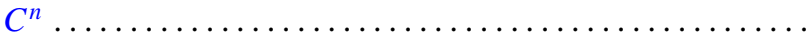

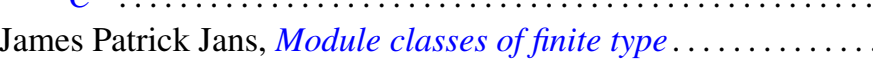

Betty Kvarda, On densities of sets of lattice points ...

H. Larcher, A geometric characterization for a class of discontinuous groups of linear fractional transformations .

John W. Moon and Leo Moser, Simple paths on polyhedra .

T. S. Motzkin and Ernst Gabor Straus, Representation of a point of a set as sum of

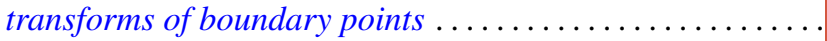

Rajakularaman Ponnuswami Pakshirajan, An analogue of Kolmogorov's three-series theorem for abstract random variables ...............

Robert Ralph Phelps, Čebyšev subspaces of finite codimension in $C(X)$...

James Dolan Reid, On subgroups of an Abelian group maximal disjoint from a given subgroup ...

William T. Reid, Riccati matrix differential equations and non-oscillation criteria for associated linear differential systems .................

Georg Johann Rieger, Some theorems on prime ideals in algebraic number fields ...

Gene Fuerst Rose and Joseph Silbert Ullian, Approximations of functions on the

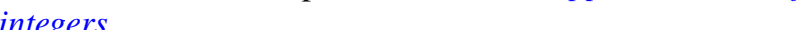

F. J. Sansone, Combinatorial functions and regressive isols . . . . . . . . . . 703

Leo Sario, On locally meromorphic functions with single-valued moduli . . . . . . . 709

Takayuki Tamura, Semigroups and their subsemigroup lattices.

Pui-kei Wong, Existence and asymptotic behavior of proper solutions of a class of second-order nonlinear differential equations . . ........... 